\title{
Protée
}

\section{Le temps donné au regard. Enquête sur la réception de la peinture}

\section{Jean-Claude Passeron et Emmanuel Pedler}

Volume 27, numéro 2, 1999

La réception

URI : https://id.erudit.org/iderudit/030563ar

DOI : https://doi.org/10.7202/030563ar

Aller au sommaire du numéro

\section{Éditeur(s)}

Département des arts et lettres - Université du Québec à Chicoutimi

ISSN

0300-3523 (imprimé)

1708-2307 (numérique)

Découvrir la revue

Citer cet article

Passeron, J.-C. \& Pedler, E. (1999). Le temps donné au regard. Enquête sur la réception de la peinture. Protée, 27(2), 93-116. https://doi.org/10.7202/030563ar
Résumé de l'article

La sociologie de la réception artistique essaie de tirer toutes les conséquences du fait - souvent rappelé mais plus rarement exploré - que les oeuvres picturales ou musicales comme les oeuvres littéraires n'existent et ne durent que par l'activité interprétative de leurs publics successifs. Prenant appui sur une observation quantifiée réalisée dans un musée français (Aix-en-Provence), cette enquête multiplie les indicateurs des comportements et des déambulations des visiteurs dans leur face-à-face avec des toiles singulières. Rapportés aux groupes qu'ils caractérisent, les classements et les catégorisations des oeuvres sont opérés a posteriori à partir du traitement dont celles-ci sont l'objet : les déterminations et les composantes du goût que révèle cette enquête s'écartent tout autant des catégorisations savantes de l'histoire de l'art que des découpages de la sociologie des consommations culturelles. 


\section{LE TEM PS DO N NÉ AU REG ARD EN QUÊTE SUR LA RÉCEPTION DE LA PEINTURE ${ }^{1}$}

JeAn-Claude Passeron et Em manuel Ped ler

\section{HYPOTHĖSES, MÉTHODES, INDICATEURS ET TERRAIN}

La sociologie de la réception artistique essaie de tirer toutes les conséquences du fait - souvent rappelé mais plus rarement exploré - que les œuvres picturales ou musicales comme les œuvres littéraires n'existent et ne durent que par l'activité interprétative de leurs publics successifs. S'attachant à décrire les "pactes» de réception artistiquequi caractérisent époques et publics, une sociologie dont les observations et les mesures se centrent sur le sort réservé à des œuures singulières porte en fait sur un tout autre objet que celui de la sociologie de la consommation culturelle dont les quantifications génériques occupent aujourd'hui la plus grande place dans les enquêtes menées en sociologie de la culture. Une sociologie de la réception s'intéresse d'abord aux actes sémiques de l'expérience esthétique (littéraire, picturale ou musicale), qu'elle vise à distinguer par des indicateurs objectifs - ce en quoi elle relève pleinement de la sociologie d'enquête.

\section{Sociologie et iconologie}

Dans la recherche portant sur des arts non verbaux, trois principes de méthode sont indispensables pour soumettre à l'observation le projet d'une «esthétique de la réception": (a) le principe de perceptibilité, qui impose à l'enquête de n'attribuer à une œuvre que les aspects de l'œuvre qui ont été perçus par des publics réels, (b) le principe de spécificité, qui impose de tirer, dans la technique d'observation, les conséquences méthodologiques de la différence entre l'activité sémiotique qu'implique l'interprétation des images, lorsqu'on la compare à l'interprétation du déroulement d'un texte, aux diégèses filmiques ou aux compositions musicales, et (c) le principe de singularité qui impose de toujours prendre pour objet d'analyse la réception d'œuvres particulières, afin de pouvoir mettre le comportement matériel et symbolique des spectateurs en rapport avec la structure iconique de chaque œuvre décrite en sa singularité2.

L'enquête sur des publics réels est donc exigible, avec ses contraintes d'échantillonnage et de standardisation des données, si l'on veut utiliser pleinement les ressources du raisonnement comparatif qui ne prend toute sa force explicative qu'avec le recours à des techniques de mesure prêtant à traitement 
statistique. Dans le rapport à des œuvres plastiques, ou à des images, de nombreuses situations de contact entre un public et un matériel iconique peuvent prêter à enquête: le feuilletage ou le stockage de reproductions comme la décoration de l'habitat, la rencontre des images publicitaires ou de l'affiche, l'entrelacs du texte et de l'image tel que l'aménagent les différents médias ou genres (livres illustrés, catalogues, bandes dessinées, sans compter toutes les associations du verbe et de l'icône fixe ou diégétique). Si l'on a choisi ici, pour préluder à d'autres enquêtes testant le regard porté sur la peinture, de rôder une méthodologie de l'observation quantifiée sur un public de visiteurs de musée - objet souvent sollicité par l'enquête sociologique -, c'est que l'espace et le contenu de l'offre muséale présentent l'intérêt méthodologique de maintenir constantes quelques variables du rapport entre des « regardeurs" et une collection de toiles. Un musée fixe en effet quelquesunes des conditions de l'expérience artistique des images: l'offre de peinture et son volume, l'espace du visionnement et la disposition des toiles dans leur environnement, le caractère formellement ouvert à tous et, en tout cas, public de la rencontre avec des tableaux. La situation de visite d'un musée procure à l'observation sociologique un public de pratiquants, appréhendé sur les lieux mêmes de sa pratique. En trop de "réflexions esthétiques» ou d'«analyses iconologiques" - qu'il s'agisse de peinture ou de littérature -, le spectateur ou le lecteur qu'invoque l'analyse formelle ou sémiotique des messages artistiques n'est autre qu'un «destinataire» déduit ou supposé à partir de quelques «marques» de l'image ou du texte. Pour la réception littéraire déjà, le «lecteur implicite», attendu par le texte, gagnerait à être plus souvent confronté au lecteur réel saisi dans l'exercice empirique de son activité de lecture. Que dire alors de la «lecture d'images»! Codes, langues, syntaxes et autres catégories linguistiques deviennent d'un maniement ambigu. L'enquête dissuade, en tout cas, de s'en tenir aux «variations imaginaires" par lesquelles les théoriciens de l'art se donnent comme allant de soi un lectorat ou un public de spectateurs dont les caractéristiques alléguées ne reposent que sur l'universalisation arbitraire des impressions privées du commentateur.

L'obligation de prendre en compte la spécificité des signifiants d'un art non verbal, spécificité définie par une texture sémiotique et une organisation temporelle différentes selon les messages, engage à une certaine méthodologie. La défiance vis-à-vis des «discours d'accompagnement", produits de l'exercice du commentaire lettré, inégalement maitrisé par les différents groupes socioculturels, est ici plus exigible qu'ailleurs, si l'on veut restituer à l'enquête l'ensemble des réceptions artistiques telles qu'elles s'effectuent dans leur diversité sociale. Ce constat a suggéré de recourir, dans un «observatoire», celui d'un musée, à une ethnographie quantitative. Par ses contraintes de standardisation, une telle grille est en effet seule capable de procurer au traitement des données un matériel comparatif, en privilégiant les aspects directement observables des actes sémiques non verbaux, par exemple les durées, les rythmes et les formes du visionnement des tableaux. Il en irait de même pour les découpages et les formes d'identification des œuvres musicales. Le projet de recourir à des indicateurs objectifs de la perception esthétique distingue donc la sociologie de la réception des œuvres d'une sociologie des représentations de l'art qui trouve l'essentiel du matériel qu'elle interprète dans les discours d'accompagnement de l'expérience esthétique.

\section{Le temps de la contemplation}

Parmi ces indicateurs, on a été amené, dans une enquête conçue comme une enquête-pilote, à privilégier la mesure des arrêts devant les tableaux et $d u$ temps consacré à leur visionnement. Ce choix de méthode semblera peut-être relever du parti pris; il n'est en fait que le choix d'une méthodologie conçue aux fins d'explorer toutes les possibilités descriptives d'un tel indicateur, qui présente l'avantage de procurer au traitement des données une "variable quantitative» au sens strict (d'où possibilité de calcul de moyennes, d'intervalles, de coefficients). L'idée que le décompte 
des arrêts devant chacun des objets d'une collection exposée aux yeux d'un public permet de saisir et, par la comparaison, d'analyser des intérêts ou des curiosités différentielles, a déjà été largement utilisée dans des enquêtes de muséologie ou de psychologie expérimentale ${ }^{3}$. Mais ces enquêtes, qui fixent d'ailleurs à un seuil fort différent la définition de «l'arrêt» (de 6/10 de secondes à 5 secondes selon les auteurs), tendent presque toujours à substantialiser «l'arrêt» en l'isolant comme une "réponse» autosuffisante: elles considèrent en somme le pouvoir d'arrêter plus ou moins de sujets comme un indicateur suffisant du pouvoir d'arrêter plus ou moins longtemps ces sujets et, implicitement, comme un indicateur du pouvoir de l'œuvre d'intéresser plus ou moins fortement les spectateurs. On a voulu ici, quitte à s'imposer une technique plus exigeante de l'observation, chiffrer les longueurs de visionnement, en pariant que les deux indicateurs - le bref arrêt et la longueur de la contemplation - ne mesuraient pas le même rapport à l'œuvre, ce qui devait permettre, et a permis, de tester la comparabilité des classements opérés par chacun d'eux ${ }^{4}$. Plus généralement, c'est la multiplication des indicateurs du comportement dans un musée face à chaque tableau, telle qu'on l'a pratiquée dans cette enquête (déambulation, retour, lecture de notice, prise de distance, regard jeté en passant), qui permet de répondre à la question que pose le caractère aveugle d'un indicateur comportemental comme l'arrêt devant un tableau: quelle est la signification psychologique et surtout artistique de ce que l'on mesure? On verra que la comparaison des informations fournies par divers indicateurs permet souvent de préciser descriptions et interprétations.

On objectera sans doute, sur le fond, que le temps d'arrêt devant un tableau constitue un indicateur bien trop grossier pour «indiquer» ou révéler grand-chose d'une expérience aussi complexe et subtile que l'expérience esthétique de la peinture. Le temps passé à contempler un tableau agglomère sans doute des plaisirs et des actes sémiques très différents, que sa longueur ne suffit certainement pas à séparer. Rien ne permettra jamais de savoir si le visiteur que le sociologue a chronométré longuement immobile devant la Bethsabée s'abîmait dans une contemplation «purement» esthétique, «dépourvue donc de toute motivation "pathologique" " au sens de Kant (Critique du jugement, Paris, Vrin, 1946), ou si sa rêverie était érotique, s'il décortiquait froidement des caractéristiques de la touche de jeunesse du peintre, s'il était en train de ruminer ses tracas financiers ou, plus simplement encore, s'il digérait un repas trop copieux. À cette objection qui court les rues et qui porterait indifféremment contre l'usage que font les sciences sociales de tous leurs indicateurs et de toutes leurs mesures, il n'y a pas d'autre réponse que statistique: un corpus d'informations, pour autant que celles-ci ont été standardisées aux fins de comparaison, permet toujours, par-delà l'objection du sens commun qui collectionne les cas limites, atypiques ou erratiques, de dégager les relations tendancielles. C'est sur ces relations stochastiques que repose la connaissance différentielle et contrastive propre aux sciences de l'homme: il faut ici, comme le disait humoristiquement Boas, «se contenter de vérités à $40 \%$ "

L'expérience de l'art est chose assez complexe pour que théoriciens et philosophes qui entendent la décrire dans son ontologie, sa phénoménologie ou sa structure s'y soient beaucoup contredits. Alain, en ses Propos sur l'esthétique, disait qu'il n'y a guère à gloser sur l'effet artistique, une fois constaté le fait incontournable que certains objets ou spectacles détiennent le pouvoir d'arrêter, de "stupéfier", comme le fait «un mur cyclopéen» lorsqu'on le découvre à Mycène. Wittgenstein, en ses Conversations sur l'esthétique ${ }^{5}$, ramenait lui aussi l'analyse esthétique à l'évidence phénoménologique que le sentiment du «beau» relève d'abord de l'ordre expressif de «l'interjection», de la «mimique», et non du «jugement attributif» que l'illusion grammaticale produit dans le langage dès que nous parlons du «beau» comme d'une propriété des choses ${ }^{6}$. Autrement dit, la propriété la plus certaine qui permet de dire qu'une expérience est artistique, c'est son intensité interne. Mesurer le temps que des visiteurs de musée consacrent à chaque toile n'est donc pas la 
plus mauvaise manière de décrire objectivement l'effet que leur fait cette toile: en tout état de cause, elle leur fait dépenser le temps de la contemplation, qui est un temps privé. C'est en tout cas le temps que les visiteurs d'un musée se sentent spontanément le plus libres de dépenser ou de refuser, ne serait-ce que parce qu'ils ne le décomptent pas ou guère.

On peut en effet justifier le choix de méthode propre à cette enquête par la spécificité psychosociologique des rapports qui s'établissent chez un sujet entre la «temporalité» de sa réception et la structure sémiotique des différents types de «messages». Il semble bien que l'image, plus que tout autre message, tende à soustraire à la conscience le pouvoir de décompter le temps qu'elle accorde à l'inspection ou à la contemplation des formes, couleurs et suggestions d'un tableau, alors que - on le remarque dans les enquêtes sur la lecture - le volume de pages lues est toujours assez exactement perçu et mémorisé par le lecteur. Chaque fois que l'on peut comparer le résultat d'une mesure objective du temps donné à un tableau à ce que les contemplateurs en disent pour justifier leur admiration ou leur intérêt, c'est - cette enquête en offre de multiples exemples la mesure terre à terre qui dit plus vrai que le discours d'apparat, de convention ou de faire-valoir. En fait, le temps accordé par le regard à un tableau est ce que le regardeur contrôle et évalue le moins bien ${ }^{7}$ : excellente occasion de prendre la mesure d'une réalité non biaisée par le langage avec toutes les majorations et tous les travestissements qu'autorise son usage social.

L'originalité de cette enquête tient assurément au choix de rapporter l'essentiel des mesures et des observations faites sur les "regardeurs» à des tableaux identifiés et traités dans leur singularité d'œuvre, insubstituable par une autre œuvre. L'intérêt de la méthode fait en même temps sa lourdeur, puisqu'elle oblige à une certaine minutie technique: des conclusions statistiquement sûres appelleraient de plus vastes collections et de plus larges échantillons que ceux qui ont été utilisés dans cette première enquête, dont les résultats esquissent d'abord des hypothèses et testent des algorithmes de traitement ${ }^{8}$. Mais, sur le fond, il est certain que cette démarche est la seule capable de procurer son objet à une sociologie de la réception artistique puisqu' elle maintient ouverte, tout au long du traitement des données, la possibilitéde mettre en rapport les comportements des spectateurs avecl'analyse formelle, iconographique ou iconologique qui, par définition, ne peut s'appliquer qu'à des œuvres singulières. Ce choix n'exclut pas la possibilité de regrouper les œuvres dans des catégories de la perception artistique construites a posteriori. Mais, à la différence des questionnaires et des questionnements courants qui se contentent d'emprunter à la taxinomie commune ou savante des courants artistiques ses découpages a priori, déjà stabilisés dans le langage de tous les jours ou l'histoire de l'art, la méthode choisie subordonne la constitution de catégories descriptives à la constatation statistique que des œuvres singulières ont été regroupées objectivement par le comportement visuel de leurs spectateurs.

\section{Le sort fait à chaque tableau}

Les attitudes et les prédispositions des groupes, telles que les mesure la «sociologie des pratiques culturelles», ne sont jamais référées qu'à des œuvres elles-mêmes considérées dans leur généralité interchangeable ou, au mieux, dans leur appartenance à un «genre». Si toutes les variations du regard jeté sur un tableau étaient résumées par ces résultats, on pourrait effectivement considérer que, lorsqu'ils regardent de la peinture, les visiteurs sont porteurs d'attitudes et d'attentes qui varient selon les propriétés socioculturelles des groupes auxquels ils appartiennent, mais que ces prédispositions s'actualisent de la même manière quel que soit le tableau qu'ils ont sous les yeux, pourvu, à tout le moins, que ces tableaux appartiennent à un même «genre» culturel, à une même «catégorie» de produits. C'est là une limite que son questionnement générique marque à une sociologie de la consommation culturelle du musée, pratique dont la géographie sociale est déjà assez connue. Le propre de la sociologie de l'art consiste à se demander si l'effet propre de chaque ouvre se moule mécaniquement 
dans ces cadres et n'obéit qu'à leurs relations de dépendance ou s'il manifeste une efficacité artistique qui se compose avec les attitudes génériques dont sont porteurs les visiteurs.

Les choix méthodologiques de cette enquête découlaient précisément d'une question théorique qui visait à construire comme deux objets différents, (a) la «consommation" indifférenciée de musée et (b) l'interprétation inhérente à la réception d'une œuvre artistique. Il s'agissait en somme de se donner les moyens de séparer et comparer ce qui revient aux prédispositions culturelles des visiteurs et ce qui revient à l'effet spécifique d'une œuvre singulière: l'hypothèse soumise ici au test empirique est évidemment qu'aucun des deux modèles causaux n'est vérifié en son intégralité - ni le modèle intégralement sociologiste, selon lequel la réception des regardeurs pourrait se déduire de leurs propensions génériques quel que soit le tableau regardé, ni le modèle de l'efficacité miraculeuse de l'œuvre, selon lequel les tableaux détiendraient, chacun en sa singularité d'œuvre, le pouvoir de faire ressentir aux regardeurs les effets qu'implique son efficace propre, quelles que soient les attitudes socialement conditionnées des visiteurs. Autrement dit, cette enquête a voulu se donner les moyens de rassembler des données permettant d'explorer l'hypothèse qu'on ne retrouve pas, dans l'usage que les visiteurs et les différentes catégories de visiteurs font de chaque tableau, l'usage qu'ils font du musée en général, ou de ce musée consommé sans préférence pour un peintre ou une œuvre.

Les questions descriptives que se pose le sociologue peuvent donc être différentes selon les tableaux que regarde un visiteur. La mesure organisée selon notre dispositif d'observation peut nous apprendre quelque chose, à la fois $(a)$ à propos des tableaux qui ont le plus d'effet insubstituable sur la réception (effet artistique tendanciellement peu sensible aux variables sociologiques) et (b) à propos des prédispositions des visiteurs plus ou les moins rebelles à l'effet de réception propre d'un tableau. Pour ce faire, on se référera non seulement au nombre d'arrêts, mais surtout à la longueur du temps de visionnement consacré à chaque tableau considéré dans sa singularité. Les enquêtes qui ont porté jusqu'ici sur le pouvoir respectif des objets d'exposition et qui ont été menées dans une perspective muséologique ont tendu à caractériser le pouvoir d'arrêt d'un tableau ou d'un objet exposé en le rapportant exclusivement au nombre d'arrêts qu'il a suscité - quitte à définir de manière plus ou moins exigeante l'arrêt minimum. Dans cette perspective, on renonce à analyser «l'arrêt" par comparaison avec d'autres indicateurs puisqu'on considère le pouvoir d'arrêter plus ou moins de sujets comme un indicateur du pouvoir d'arrêter plus ou moins longtemps un même sujet. En se donnant ici une technique plus lourde afin de décompter les longueurs de stationnement devant chaque tableau, on a parié que ces deux mesures constituaient les indicateurs de deux effets différents susceptibles d'être produits par une peinture ou un objet offert au regard dans une collection. On verra en effet, par les variations du rang des tableaux qu'opèrent les deux indicateurs, que ces deux mesures ne favorisent pas les mêmes tableaux. On peut en inférer que ceux-ci ne produisent pas les mêmes effets perceptifs ou artistiques. Toute une série d'autres indicateurs du comportement muséal face aux tableaux ainsi individualisés et surtout d'autres traitements statistiques du temps donné aux tableaux par exemple le fait de pouvoir rapporter le temps d'un sujet (ou le temps moyen d'un groupe) face à un tableau déterminé au temps théorique que celui-ci lui aurait accordé, en fonction du temps qu'il a consenti au musée, s'il avait distribué ce temps global au hasard ${ }^{9}$ - permettent en principe, par l'analyse des variations de classement que déterminent ces différentes mesures, de formuler quelques hypothèses interprétatives sur le sort différent que les différents groupes de sujets font aux différents tableaux du musée.

Pour aller du plus générique au plus spécifique, on présentera d'abord ici les classements que nos mesures opèrent sur les tableaux exposés dans les deux salles de l'enquête, lorsque l'on considère l'ensemble de l'échantillon (II). Ce premier point de vue sur les tableaux, qui consiste à interroger le pouvoir propre à 
chaque tableau de faire s'arrêter et stationner plus ou moins longtemps les visiteurs sans considération de leur appartenance sociale ou de leurs caractéristiques culturelles, correspond en somme à ce que l'on pourrait appeler le point de vue marchand sur les tableaux qu'un musée soumet à un marché $d u$ visionnement. Dans ce "modèle», on instaure une expérience imaginaire de rentabilisation du fonds d'œuvres offert à l'œil par un Musée (ou par un Conservateur qui s'identifierait à la maximalisation de l'usage marchand de son offre). Il ne s'agit alors que de savoir avec précision combien chacun des tableaux offerts parvient à prélever de temps (le coût en temps concédé étant ici l'équivalent d'un paiement monétaire, virtuel ou éventuel) sur le temps global qu'un flux de visiteurs donne au musée. Pour les besoins simples d'une telle comptabilité, ce serait effectivement une solution que de classer les différents tableaux par le score global qu'ils obtiennent sur le marché du regard. Peu importerait en effet à un musée, s'il pouvait penser en tant que gestionnaire calculateur de ce qu'il fait payer aux visiteurs pour chacun des tableaux qu'il offre au regard, que tel ou tel groupe de visiteurs - les femmes plutôt que les hommes, les prolétaires ou les snobs, les passionnés d'art ou les conformistes, les gens pressés ou les nonchalants - ait classé différemment par le temps consenti les tableaux offerts à l'œil. Au total, par rapport au flux des visiteurs, il importerait seulement à ce modèle d'un musée calculateur - forme pure et extrême d'une pensée de marketing - de connaître, à toutes fins utiles, le pouvoir d'arrêt différentiel des tableaux donnés en spectacle.

Une telle analyse de la dépense en temps consentie par les visiteurs repose exclusivement sur le point de vue d'un musée indifférent à l'arbitrage que fait chaque visiteur entre le plaisir pris à un tableau et le temps global qu'il peut donner au musée. Dans une telle mesure, le score d'un tableau - qu'on le définisse par la somme des temps donnés par les visiteurs qu'il a arrêtés ou par la valeur moyenne du temps que lui a donné un visiteur - agrège des dépenses en temps consenties par des visiteurs qui ne disposaient pourtant pas du même temps global à distribuer entre les différents tableaux puisqu'ils ont cheminé plus ou moins rapidement dans le musée. Si cette inégalité des budgets-temps propres aux différents visiteurs est sans conséquence pour une comptabilité qui ne se préoccupe que de ce qu'a «rapporté» un tableau, indépendamment de la richesse ou de la pauvreté relatives des visiteurs qui ont payé sa contemplation de leur temps, elle produit un classement des tableaux dans lequel les choix des visiteurs pèsent d'autant plus ou d'autant moins qu'ils ont stationné plus ou moins longtemps dans le musée en fonction de leurs emplois du temps, de leurs habitudes ou de leurs contraintes externes. Il faut donc, pour les besoins d'une analyse rigoureuse de la demande artistique, essayer de neutraliser cette inégalité des budgets-temps afin de serrer de plus près goûts et préférences des visiteurs: ici on a pondéré le temps donné à un tableau par chaque visiteur par le temps moyen que celui-ci a consacré aux différents tableaux devant lesquels il s'est arrêté.

Résultat négatif mais important pour isoler l'existence d'un pouvoir de singularité propre à chaque tableau: les résultats obtenus grâce à ce raffinement de l'analyse obligent à constater la superposition presque complète $d u$ classement des tableaux opéré par les temps moyens, qu'ils soient "bruts» et "pondérés». Le palmarès des tableaux présentés dans les salles $\mathrm{A}$ et $\mathrm{C}$, selon ces deux indicateurs, est le même, puisque les permutations, d'un bout à l'autre du classement, ne portent jamais sur plus de deux ou trois rangs; le classement selon les temps «bruts» ou "pondérés» est exactement le même en haut comme en bas du classement. Ce parallélisme des classements opérés par les temps moyens «bruts» et «pondérés» est d'autant plus remarquable que les classements opérés par d'autres indicateurs du comportement muséal face à chacun des tableaux (arrêts, nombre de notices lues, nombre de retours) produisent, eux, de fortes perturbations de rang ${ }^{10}$.

On réserve ici à un deuxième temps l'analyse des classements que les mesures de l'enquête opèrent entre chacun des 32 tableaux lorsqu'on considère les 16 groupes que notre enquête permet de séparer (III). 
Ce point de vue, fondé sur la différenciation interne du public de visiteurs, permet alors de décrire non seulement des goûts ou des dédains spécifiques à chaque groupe, mais aussi l'effet propre de certains des 32 tableaux ou de l'association de plusieurs d'entre eux selon que cet effet varie ou non entre les groupes. On opère ainsi, si l'on veut, un tri des singularités par les singularités - visiteurs et tableaux - puisqu'on apprend quelque chose des œuvres par la connaissance sociologique de ceux qui les choisissent et du goût des visiteurs par la connaissance iconologique de ce qu'ils choisissent.

\section{LE TEMPS DONNÉ AUX TABLEAUX}

1. Le musée, le capital scolaire et les cuvres

Avant même d'en venir aux mesures particulières, l'ethnographie quantitative qui permet, dans cette enquête, d'individualiser le parcours de chaque sujet de l'échantillon, fait ressortir un effet paradoxal du niveau de diplôme sur le comportement de visite d'un musée. Depuis L'Amour de l'art ${ }^{11}$, la sociologie de la fréquentation du musée s'est habituée à retenir le niveau d'instruction comme variable principale, parfois unique, de toute variation d'un comportement culturel, en tout cas comme une variable dont l'action serait «linéaire». Or, si l'on s'intéresse à la mesure la plus globale du coût consenti par les visiteurs pour regarder de la peinture - ici le temps qu'ils consacrent au musée, entre l'entrée et la sortie - les variations du temps donné au musée en fonction des différents niveaux de diplôme révèlent, comme en toute enquête sur une pratique culturelle, une corrélation statistique. Mais elle est en ce cas d'une forme paradoxale, dès lors que, comme sur notre échantillon bien fourni en hauts diplômes, on a pu distinguer au sein des études supérieures deux niveaux (études courtes; études longues). Comme on peut le voir dans le tableau qui suit, les sujets les moins diplômés se retrouvent dans la zone moyenne de l'indice mesurant le temps donné au musée, tandis que les diplômés moyens de l'enseignement supérieur sont représentés plus que proportionnellement dans la zone haute de l'indice du temps donné au musée.
Tableau 1

Le temps donné au musée (indice) selon le diplôme

\begin{tabular}{|c|c|c|c|c|}
\hline Diplôme* $^{*}$ Indice & $\begin{array}{c}\text { Inférieur } \\
\text { à } 30^{\prime}\end{array}$ & $\begin{array}{c}\text { Entre } 30^{\prime} \\
\text { et } 47^{\prime}\end{array}$ & $\begin{array}{c}\text { Au-dessus } \\
\text { de 48' }\end{array}$ & Total \\
\hline $\begin{array}{l}\text { études élémentaires } \\
\text { et secondaires }\end{array}$ & $23 \%$ & $51 \%$ & $26 \%$ & $100 \%$ \\
\hline $\begin{array}{l}\text { études supérieures } \\
\text { courtes }\end{array}$ & $28 \%$ & $39 \%$ & $33 \%$ & $100 \%$ \\
\hline $\begin{array}{l}\text { études supérieures } \\
\text { longues }\end{array}$ & $40 \%$ & $36 \%$ & $24 \%$ & $100 \%$ \\
\hline Ensemble & $31 \%$ & $41 \%$ & $28 \%$ & $100 \%$ \\
\hline
\end{tabular}

Ce sont donc, paradoxalement, les sujets les plus hautement diplômés qui sont proportionnellement les plus représentés au niveau faible de l'indice (ce que souligne ici en gras de la plus forte tendance par colonne). Autrement dit, en allant des temps les plus brefs passés dans le musée aux temps les plus longs, on rencontre d'abord (parmi les plus représentés) les sujets les plus diplômés, puis les moins diplômés avant de rencontrer les sujets moyennement diplômés qui sont ceux qui passent le plus de temps dans le musée.

En caractérisant les visiteurs par le temps moyen donné au musée par catégorie de diplômes, on retrouve le même ordre inattendu. L'ordre croissant des temps moyens ordonne les diplômes dans l'ordre 3, 1,2. Cette structure récurrente est bien celle qui caractérise en fonction du diplôme le temps global que les visiteurs donnent au musée. On voit directement sous une forme graphique cette relation paradoxale sur un histogramme: il faut désordonner l'ordre scolaire pour que l'histogramme présente une pente régulière correspondant à l'augmentation du temps donné au musée ${ }^{12}$. (Voir le schéma 1 à la page suivante.)

Les habitudes scolaires qui engendrent la docilité envers les hiérarchies culturelles et réactualisent les disciplines mentales enseignées par l'École, doivent leur efficacité à la longue durée de cette influence. Mais efficacité ou marquage ne veut pas dire action mécanique et régulière. La relation entre la hiérarchie des diplômes ou des longueurs d'étude et ses effets se

\footnotetext{
* Le découpage des niveaux de diplôme correspond ici aux cursus français; on a distingué une formation (1) inférieure au bac, (2) située entre bac et bac+3, (3) égale et supérieure au bac+4. Nous conservons ce découpage dans la suite de ce texte.
} 
Schéma 1

L'histogramme du temps donné au musée selon le diplôme (ordonnée: temps moyen en secondes)

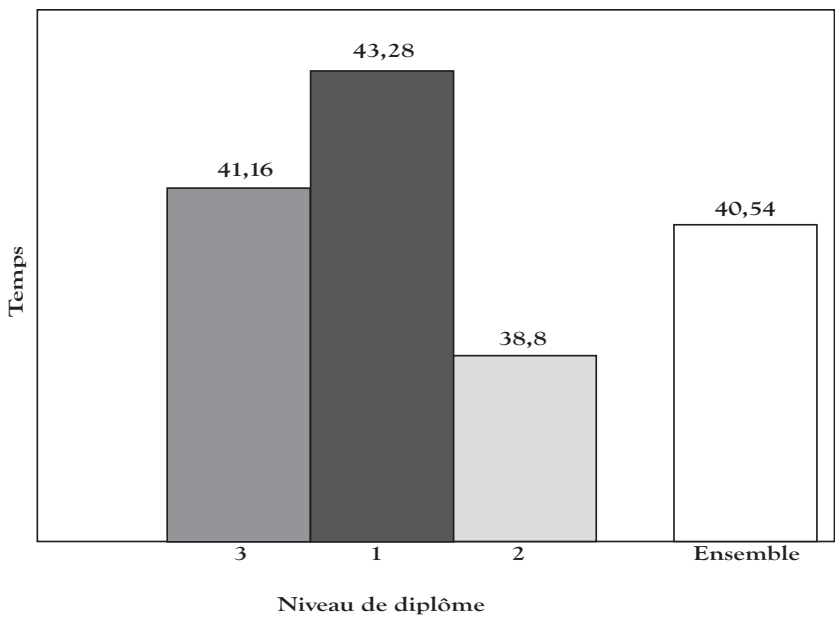

trouve, selon les effets qu'on analyse, prendre tantôt la forme "classique", tantôt la forme "paradoxale": précieuse différence qui peut renseigner sur le sens d'une pratique culturelle.

On retrouve dans cette enquête l'effet paradoxal - ou non linéaire - du niveau de diplôme pour un grand nombre d'indices, qu'il s'agisse du temps moyen par tableau regardé, du temps consacré aux deux ailes du musée, de comportements déambulatoires, ou encore de préférences pour des toiles singulières. L'effet classique - linéaire - apparaît sur d'autres comportements muséaux: l'interprétation s'en trouve changée.

2. Le succès des œuvres: le classement des tableaux en fonction du sort que lui a fait l'ensemble de l'échantillon

L'indicateur le plus direct du coût psychologique et symbolique que les visiteurs consentent à chaque tableau est évidemment le temps de visionnement qu'ils y consacrent et que cette enquête a essayé de mesurer aussi scrupuleusement que possible (par le temps «brut» ou le «temps pondéré») sur la base d'un chronométrage opéré par un enquêteur suivant discrètement un visiteur. Cet indicateur révèle, sur les 32 tableaux des deux salles où ont été conduites les observations quantifiées, une forte dispersion des temps moyens qui hiérarchisent les différents tableaux. Parallèlement, l'observation ethnographique a rencontré sous une forme gestuelle ou déambulatoire cette même variabilité du traitement réservé aux tableaux de l'exposition selon les visiteurs.

Mais le sceptique en matière d'enquête sociologique sur l'art peut encore supposer que la distribution des temps de regard sur les tableaux d'une exposition pourrait bien ne pas différer de l'usage visuel que des chalands font aléatoirement d'un étalage dans leur pratique d'exploration ou de promenade. Dans un tel modèle, qui n'est autre que celui du shopping, l'attention distraite qu'un visiteur donnerait en plus à un tableau, un autre la lui donnerait en moins et, tout compte fait, on pourrait s'attendre à ce que les tableaux obtiennent un traitement proche d'une distribution au hasard du temps de visionnement. À quelques produits-vedettes près, l'offre d'images serait ainsi consommée par la majorité des visiteurs sur le mode du "picorage». Ce modèle du pecking est-il vérifié? Nullement. Au travers de notre mesure, même limitée à deux salles d'un musée, on voit au contraire, en considérant les temps moyens de visionnement consacré à chaque tableau (quotient de la somme des stationnements de plus de trois secondes devant un tableau par le nombre de visiteurs ayant effectué un tel arrêt, pour le temps moyen «brut» 13 ), que cette mesure produit un véritable palmarès. Les scores des tableaux les plus regardés sont nettement détachés par rapport aux suivants, appelant ou suggérant ainsi des questions iconographiques ou iconologiques. La simple lecture du Tableau 2 laisse en effet perplexe et les questions qu'elle ouvre, par ses résultats agrégés, conduiront aux analyses de la troisième partie de ce texte.

Les sept tableaux figurant en tête du palmarès des temps de visionnement ont un temps moyen qui, par le dénivelé des valeurs, individualise l'effet qu'ils ont produit sur un flux de visiteurs. Ces sept tableaux sont tous situés au-dessus du temps moyen consacré à un tableau par l'ensemble de l'échantillon. 
Tableau 2

La distribution décroissante des temps moyens «bruts» consacrés aux 32 tableaux des salles A et C

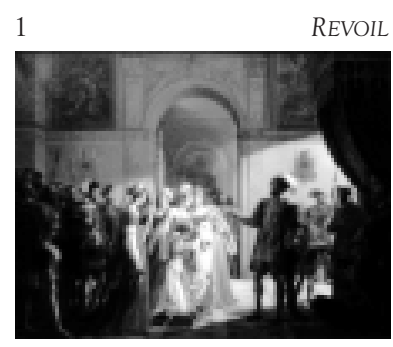

Tb9

$23,6 ”$

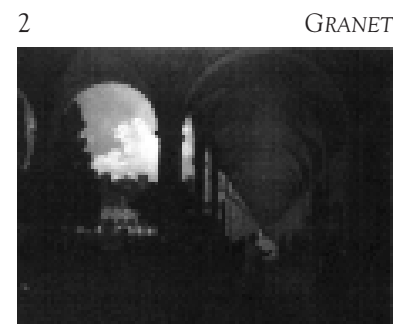

Tb5

$18,8 ”$
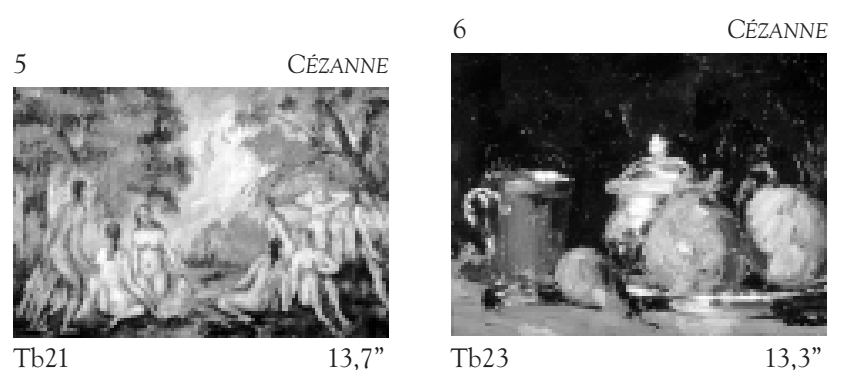

10
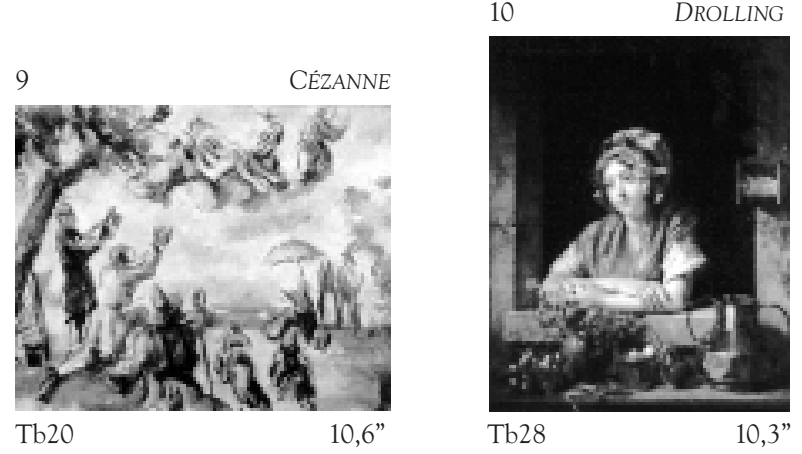

13

VAN BEEST

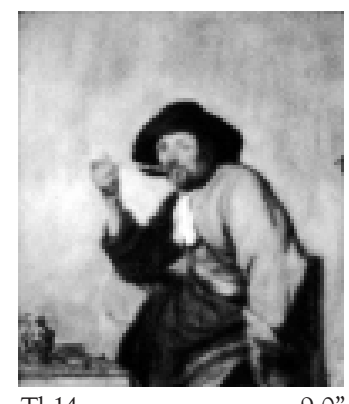

$\mathrm{Tb} 14$

14

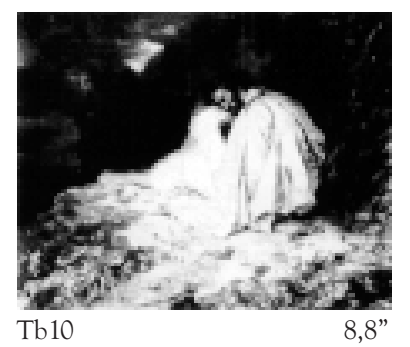

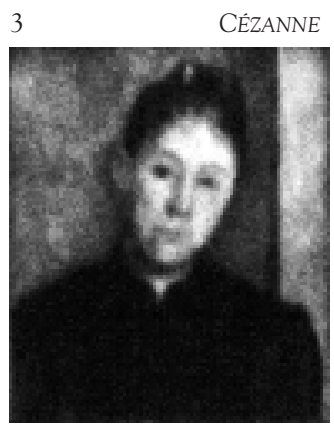

$\mathrm{Tb} 22$

15,2 "
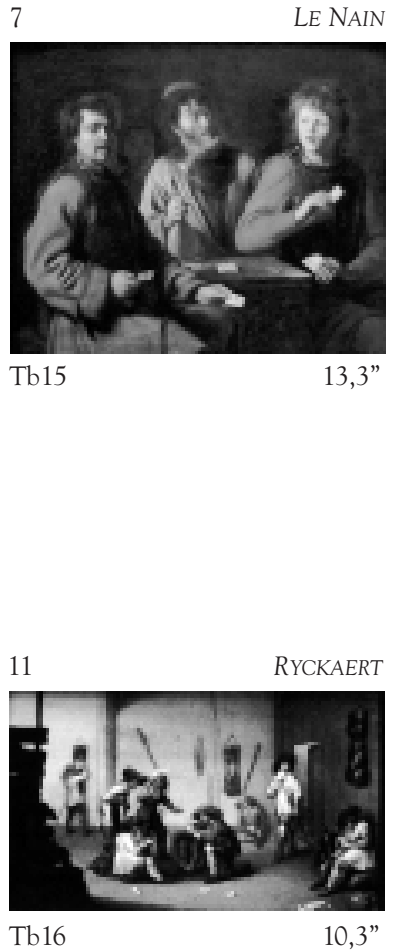

8

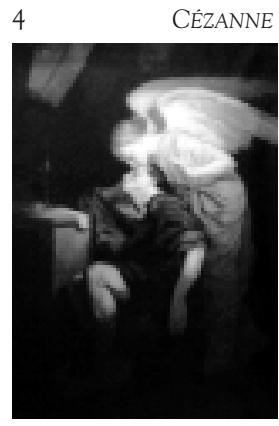

Tb31

$13,9 "$
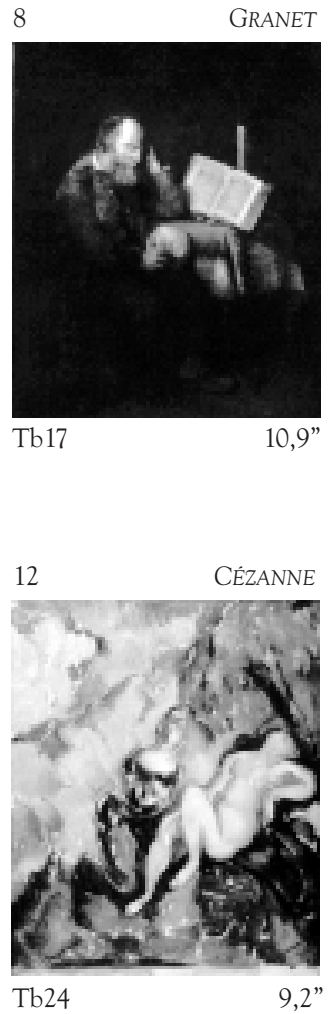

15

PEYRON

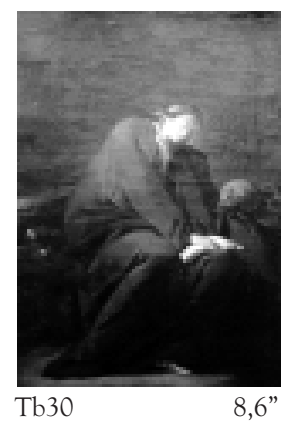

16

TENIERS

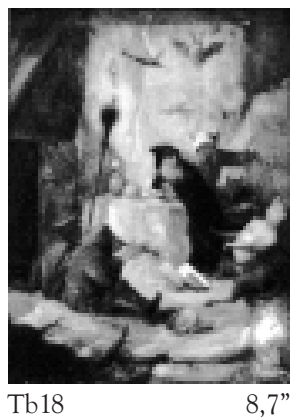




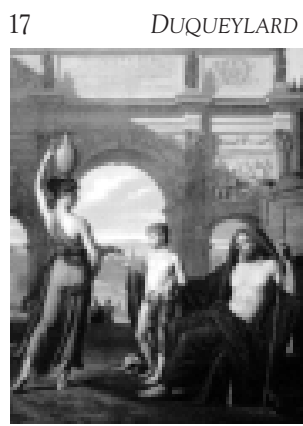

Tb2
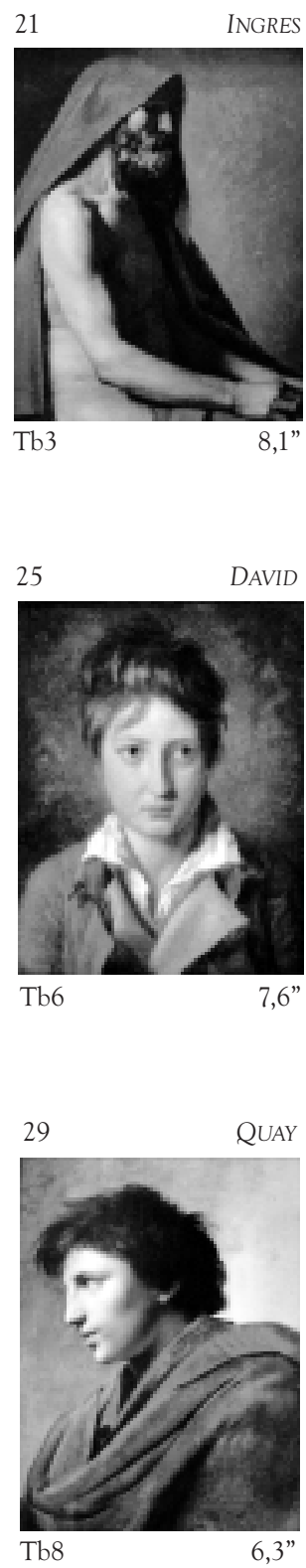

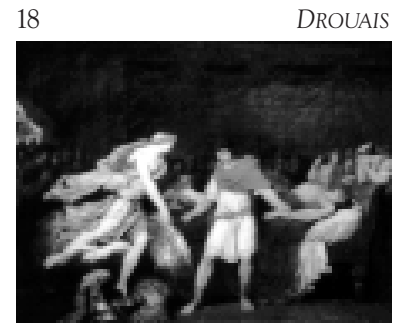

Tb4
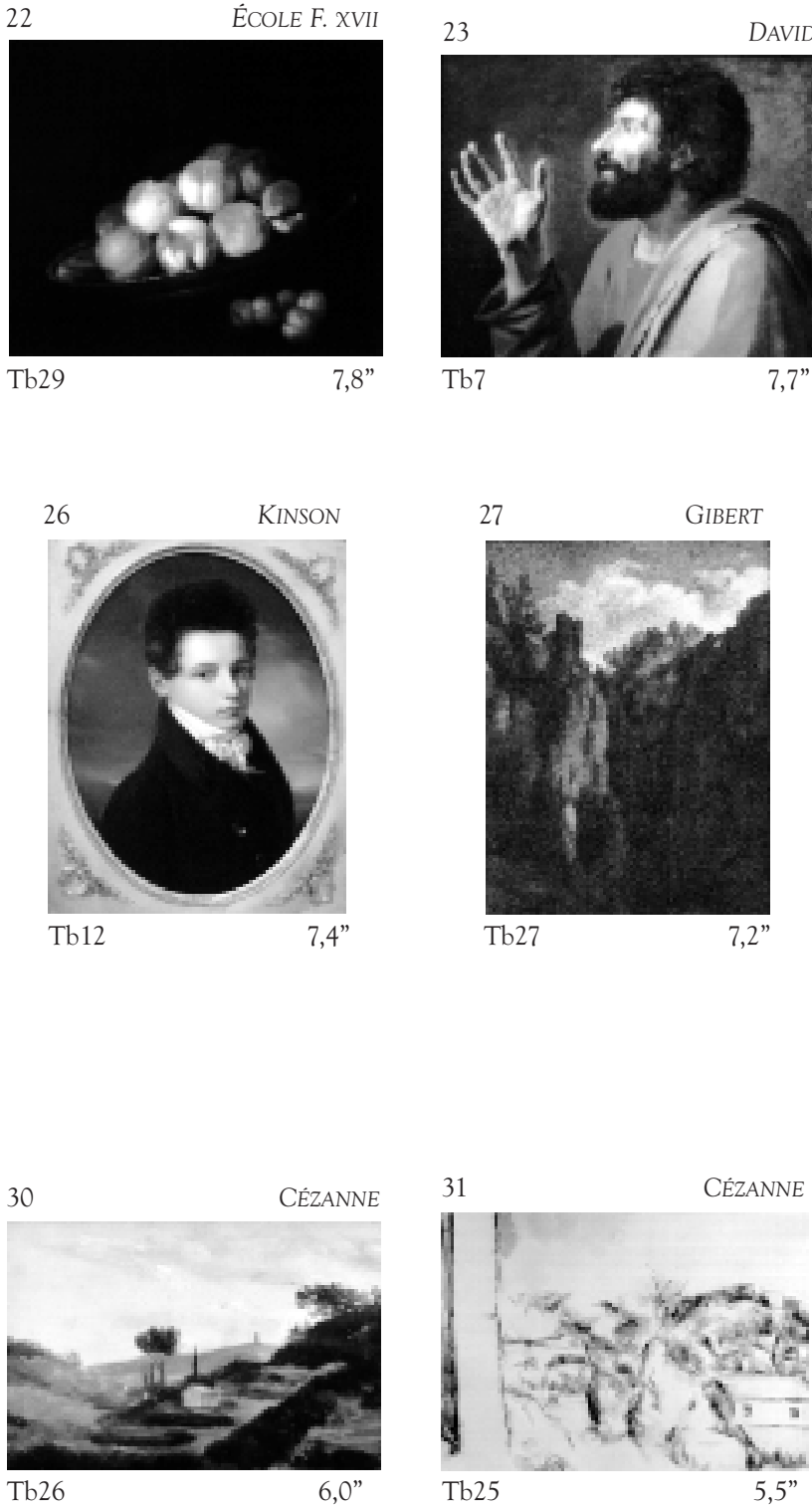

Tb1

8,1"

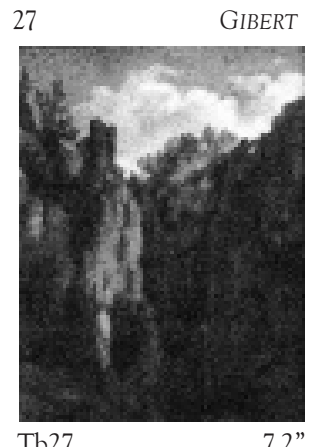

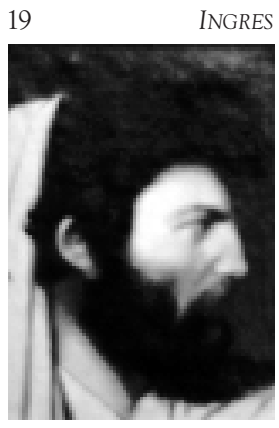
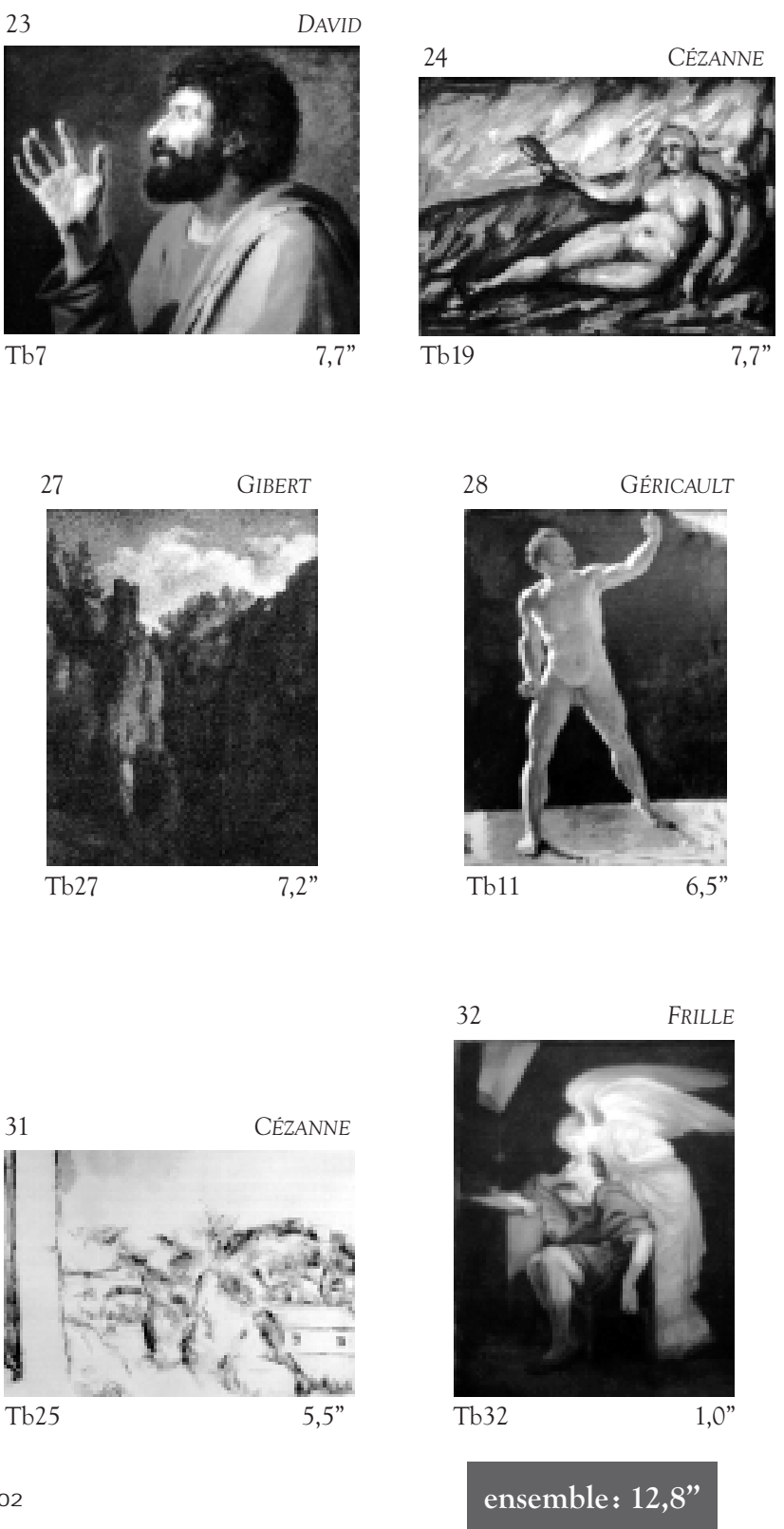
Schéma 2

Implantation des tableaux dans les salles $\mathrm{A}$ et $\mathrm{C}$
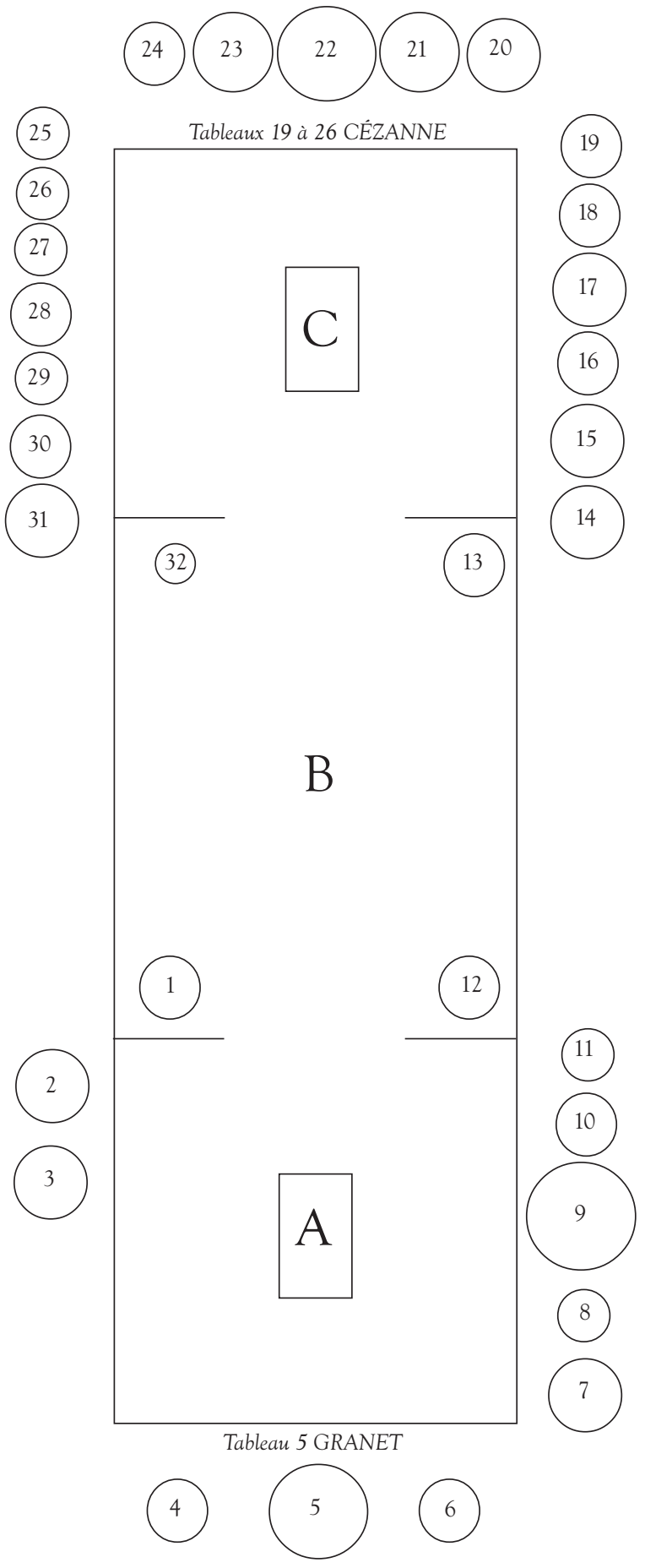

Les pastilles qui entourent le numéro des tableaux ont une surface proportionnelle aux temps moyens de l'arrêt qu'ils ont suscité de la part de l'ensemble de l'échantillon
3. La convergence des classements opérés par le comportement muséal des visiteurs

A. L'hétérogénéité du goût dans les choix majoritaires On voit se rassembler sur le Tableau 2, par

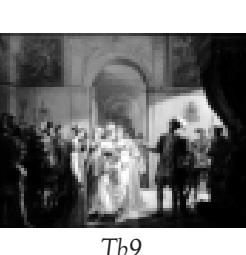
l'attention qui leur est majoritairement accordée dans une sorte de «classement général", à la fois les Cézanne les plus caractéristiques proposés par le Musée Granet (n²2, Portait de

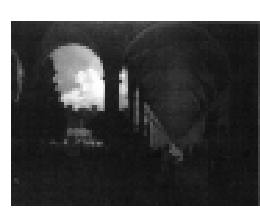
Madame Cézanne; no21, Les Baigneuses et n'23, Nature morte: sucrier, poires et tasse bleue), mais aussi, et encore mieux placées, deux toiles qui sont d'une notoriété
$\mathrm{Tb} 5$

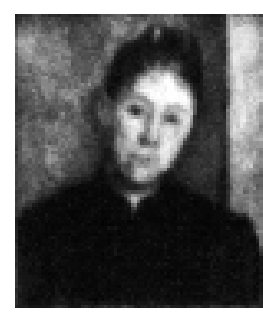

$\operatorname{Tb} 22$

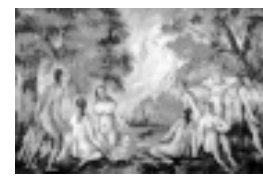

$T b 21$

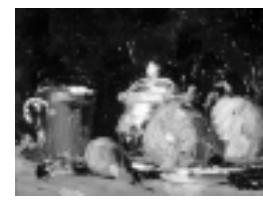

Tb23 bien moindre en même temps que d'une facture également éloignée de toutes les formes d'art contemporain. De surcroît, ces deux outsiders diffèrent entre eux tant sous le rapport du style ou de la touche que par l'appartenance du mieux classé à la peinture d'histoire la plus traditionnelle et, partant, parla familiarité culturelle ou érudite qu'ils appellent. Il s'agit du no9 (Revoil: François I ${ }^{e r}$ faisant chevalier son petit-fils François II ) et du no 5 (Granet: Le Cloître de la chartreuse Sainte-Marie-des-Anges à Rome).

Même si la variable de la taille ou du remplissage du tableau joue ici un rôle, nos mesures

empiriques interdisent certainement de conclure que ce facteur ${ }^{14}$ suffit à porter en tête, en concurrence avec des Cézanne, une peinture d'histoire de Revoil, laquelle devance même, par le temps moyen de visionnement qu'elle a obtenu, une composition tout aussi travaillée de Granet et bien plus ample. En effet, la toile de Granet avec plus de $5 \mathrm{~m}^{2}$ en impose visuellement davantage que les $2 \mathrm{~m}^{2}$ de celle de Revoil 
sans pour autant lui ravir une première place qui se trouve attestée par le temps moyen «brut» (où le Revoil, avec 23,6”, se situe au double du temps moyen), mais aussi par le temps moyen «pondéré». En outre, une troisième toile dans les salles A et $\mathrm{C}$ est, elle aussi, d'une taille analogue à celle du Revoil; c'est une peinture à l'antique de Duqueylard ( ${ }^{\circ} 2$, Bélisaire et son guide), qu'il faut pourtant aller chercher en milieu des classements, quel que soit le critère classificateur que l'on retienne (17 et $18^{\mathrm{e}}$ rangs aux temps, $24^{\mathrm{e}}$ rang au nombre d'arrêts, 19 e rang par la lecture des notices et 22 e rang au nombre des retours).

Le classement modeste d'autres toiles de ces

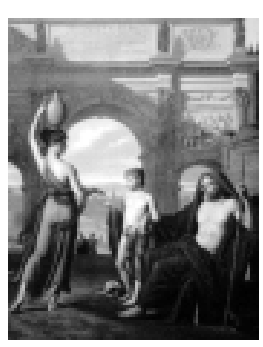
mêmes salles, qui proposent pourtant des scènes à personnages multiples dont l'interprétation narrative requerrait aussi une exploration attentive, montre, par différence, que les deux tableaux (n०9 et n०5) favorisés par l'attention des visiteurs offrent à la perception picturale des caractères communs qui semblent jouer un rôle récurrent dans l'attention qu'un tout-venant de visiteurs de musée accorde à des toiles. Il existe sans doute, pourrait-on dire en paraphrasant Aloïs Riegl, une «kunstwollen du spectateur» en étendant ainsi au «récepteur» le concept que Riegl avait forgé pour les «créateurs", une volonté ferme d'entrer dans le monde de l'art, même si elle est dépourvue des repères et des savoirs érudits, des accoutumances formelles, qui arriment chez le spécialiste ou l'amateur la perception esthétique sur des structures fines de l'œuvre. Cette "volonté» du regardeur d'avoir un plaisir spécifique se guide sur les seuls repères plastiques capables de le réassurer en lui assurant qu'il choisit un objet «méritant» d'être goûté picturalement. Dans cette tâche d'identification des tableaux qui valent la peine d'être perçus comme objets picturaux, se trouvent privilégiés les marqueurs forts d'une facture plastique capables d'attester, y compris avec redondance, l'appartenance d'une peinture particulière à la Peinture. Tout ce qui dans la composition, le traitement des détails, le fini, voire le léché, la richesse ou la rareté tant du matériau pictural que de l'objet représenté, dans la dignité ou la solennité des scènes (par opposition à l'anecdotique, au comique ou au familier), la virtuosité dans la maîtrise des difficultés surmontées au profit de l'effet illusionniste (perspectives rares, lumières, grain, reflets, sfumato) garantit et désigne une peinture comme "vraie peinture» - et cela indépendamment de la connaissance par ouï-dire des hiérarchies légitimes ou savantes - aboutit à privilégier les tableaux saturés de ces qualités directement reconnaissables à l'œil du profane. De tels tableaux sont rassurants parce qu'ils exorcisent le risque de l'erreur sur l'objet: on s'expose dès qu'on élit un objet comme objet de plaisir artistique. Le risque est évidemment de se ridiculiser devant d'autres spectateurs en admirant comme objet d'art ce qui ne l'est pas.

C'est sans doute, nous le supposons, cette composante de l'expérience artistique commune, que rien ne permet de placer hors expérience artistique ou de caricaturer comme un faire-semblant social dépourvu de tout retentissement interne, qui explique la performance du Revoil dans cette enquête. On a là le type-idéal d'un tableau capable de fonctionner comme un symbole plénier de la peinture pour ceux, les plus nombreux, qui veulent d'abord trouver dans une peinture le répondant autorisé du plaisir spécifique qu'ils prennent à une imagerie travaillée. Type-idéal que le classement des toiles permet de distinguer de celui qu'incarnent les toiles ou reproductions de "grands peintres", symboles éclatants de la peinture: tableaux ou peintres dont la légitimité nominale est connue (ou reconnue par ouï-dire), que ce soit sous les espèces des grands noms de la peinture classique ou des monstres sacrés de l'art contemporain.

\section{B. Autres convergences des classements}

Le cas des tableaux dédaignés: les "études» versus les œuvres Un groupe de cinq tableaux (Tb11, Tb12, Tb8, Tb6 et Tb3), qui figurent toujours aux derniers rangs, quel que soit l'indicateur retenu, contient trois toiles signées de noms connus, Géricault (Étude d'Académie), 
David (Première Idée pour le vieil Horace $d u$ «Serment des Horaces") et Ingres (Étude d'homme, assis de profil). Ce n'est sans doute pas un hasard s'il s'agit dans les trois cas d' "études" désignées comme telles par leur titre et identifiables par leur facture comme par l'absence d'intrigue iconographique. On est ici aux antipodes de la saturation d'un objet peint en signes extérieurs de l'appartenance à la peinture au sens plénier, dont nous venons de voir avec le Revoil le rôle qu'elle jouait pour la plus grande partie d'un public de visiteurs de musée. En tous cas les études ne sont pas elles-mêmes prédésignées par une notoriété d'œuvre: c'est bien l'individualité d'une oeuvre, reconnue ou attendue comme exemplaire singulier de la peinture, plus que le nom propre du peintre qui fait frayage de l'attention. Ceci va en sens inverse de la représentation commune de «l'effet de légitimité» qui, en sa forme aplatie et vulgarisée, porte à ne voir dans l'efficacité de la légitimité culturelle qu'un effet mécanique de la notoriété des noms d'auteur.

\section{Le regroupement des tableaux en fonction de caractéristiques $d u$ "sujet»: le "vivant» et "l'inanimé»; «l'intemporel» et le «narratif»}

L'examen des palmarès suggère aussi que le mauvais classement des tableaux relevant de certains genres, par exemple le paysage ou la nature morte - et cela bien avant le décrochage du grand public par rapport aux formes non figuratives de l'art contemporain -, isole dans l'intérêt porté à des tableaux une composante récurrente de l'expérience picturale: il y a en toute expérience artistique (n'en déplaise à Kant et à sa théorie du "pathologique» dans l'art) un «retentissement» propre aux stimulations affectives de l'œuvre qui reste assez étranger aux matières et aux structures formelles d'une peinture et qui renvoie par exemple (dans l'art figuratif) au plaisir actif de reconnaitre et d'interpréter gestes et expressions de la vie. Cette composante, dont le poids dans les goûts effectivement professés est assurément fort variable selon les groupes socioculturels, mais aussi selon les périodes historiques, définit un plaisir qui se satisfait plus facilement dans la représentation de l'animé que dans celle de l'inanimé et, plus précisément encore, dans les mises en intrigue ou en scénario du «sujet», potentialités ou manifestations de «vie» qui regroupent ici dans le milieu du palmarès de nombreuses scènes de genre ou des portraits expressifs. On aperçoit cette demande, dans notre enquête, sous une forme grossie avec les choix qui modèlent les orientations majoritaires vers le narratif ou l'anecdotique. La sensibilité différentielle des groupes à cette composante, qui, au total, pèse toujours dans un échantillon tout-venant, explique que c'est surtout dans l'examen plus affiné des préférences et des dédains des différents groupes que l'on verra son action sur les 32 tableaux ${ }^{15}$.

On peut, pour systématiser l'analyse du rôle des actes sémiques qui sont à l'œuvre dans l'interprétation d'un tableau, tirer parti d'une éventuelle proximité du classement des tableaux qui sollicitent identiquement l'activité interprétante des visiteurs selon la part qu'ils font aux «sujets» ou «motifs» animés, par opposition aux sujets ou motifs inanimés, ainsi que selon le degré auquel l'image invite à restituer une scène à un récit, à une interaction entre personnages ou à la recherche d'un «avant» et d'un "après", qui impose ainsi au spectateur une attention ou une question diégétique ${ }^{16}$.

Pour tester la convergence des effets-tableaux liés à ces deux

dimensions de l'interprétation, on a donc réparti ici les 32

tableaux en 3 catégories; (I): tableaux dont le sujet présente un

ou des êtres animés et impose ou sollicite une mise en diégèse au moins minimale pour pouvoir se prononcer sur ce qu'il représente; (II): tableaux présentant des êtres animés, mais dont l'interprétation n'appelle pas cette interrogation conditionnelle ou hypothétique sur «l'avant» ou «l'après» du moment figuré par l'image ou par certains traits ou zones de l'image; (III): tableaux excluant figures animées et mouvement diégétique. Sur le lot des tableaux de notre enquête, on est ainsi amené à les répartir, pour 12 d'entre eux, dans la première catégorie (personnages multiples, scènes réalistes mythologiques, religieuses, historiques ou énigmatiques); la seconde catégorie regroupe 15 toiles, portraits pour l'essentiel, mais aussi académies et études; puisque nous exigeons, dans la première catégorie, la présence de «sujets» ou de «motifs» appelant une interprétation diégétique 
caractérisée, c'est-à-dire capable de mettre en mouvement l'interrogation du spectateur à propos d'une interaction ou d'un scénario. La troisième catégorie, qui comprend ici cinq tableaux, rassemble les natures mortes et les paysages.

Le classement de chacun des tableaux ainsi recatégorisés contribue alors à chiffrer une réussite, auprès du spectateur, de la catégorie à laquelle appartiennent ces tableaux: on peut trancher du fait qu'il y a ou non une "prime à la réception» en fonction de cette catégorisation des contenus figuratifs. On voit la différence de cette méthode avec celle, souvent utilisée, qui consiste à questionner directement les sujets à propos de catégories dont on leur propose le nom, au lieu de rechercher l'effet d'une catégorie par l'agrégation des effets propres à chacun des tableaux qu'on y fait entrer, comme dans les calculs que nous effectuons ici: le caractère clivant ou non de la catégorie constitue alors un test de la consistance perçue de la catégorisation.

Tableau 3

Succès sur l'ensemble de l'échantillon de trois catégories de tableaux ${ }^{17}$

\begin{tabular}{|c|c|c|}
\hline CATÉGORIES & SUCCĖS DES TABLEAUX & MOYENNE \\
\hline $\begin{array}{l}\text { Animation } \\
\text { et diégèse (I) }\end{array}$ & $\begin{array}{c}\text { Tb2, Tb4, Tb5, Tb9, Tb15,Tb16 } \\
\text { Tb18, Tb20, Tb21, Tb24, Tb31, Tb32 }\end{array}$ & 22,8 \\
\hline $\begin{array}{l}\text { Animation } \\
\text { sans diégèse (II) }\end{array}$ & $\begin{array}{l}\text { Tb1, Tb3, Tb6, Tb7, Tb8, Tb10, Tb11, Tb12, } \\
\text { Tb13, Tb14, Tb17, Tb19, Tb22, Tb28, Tb30 }\end{array}$ & 15,30 \\
\hline $\begin{array}{l}\mathrm{Ni} \text { animation } \\
\text { ni diégèse (III) }\end{array}$ & $\mathrm{Tb} 23, \mathrm{~Tb} 25, \mathrm{~Tb} 26, \mathrm{~Tb} 27, \mathrm{~Tb} 29$ & 10,40 \\
\hline
\end{tabular}

Le test donne donc un résultat assez clair: il y a bien, à la réception, une prime d' «intérêt» qui va à la catégorie des «sujets» et "motifs" associant figures animées et questions diégétiques $(22,8)$ et qui est encore perceptible, dans le cas des sujets animés dépourvus d'arrière-plan diégétique $(15,30)$ par rapport aux natures mortes et paysages dont le plus faible intérêt relatif $(10,40)$ prend ici tout son sens dans une catégorisation fondée sur le type d'interprétation requis ${ }^{18}$.
4. Le coup de chapeau à la notoriété et la captation involontaire du regard: les divergences fortes et structurées entre les classements

A. L'arrêt et le visionnement: Cézanne et les autres

Si les classements par le

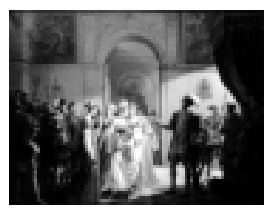

Tb9

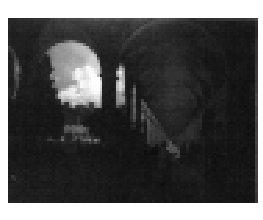

Tb5

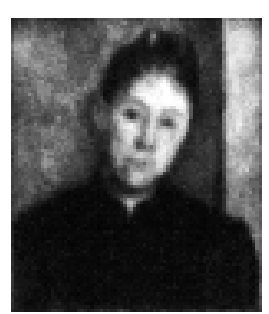

Tb22

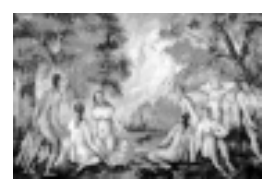

Tb21 nombre d'arrêts et par les diverses mesures du temps de visionnement convergent sur le contenu du lot de tableaux placés en tête, ils se différencient et même s'opposent par le sort inverse qu'ils font aux deux groupes de tableaux figurant dans ce lot de tête. Le classement selon les temps moyens de visionnement met au premier rang le Revoil et un Granet (Tb9 avec 23,6" et Tb5 avec 18,8") et ne laisse apparaître qu'ensuite les trois Cézanne, alors que le classement fondé sur le nombre de visiteurs qui ont concédé l'arrêt de plus de trois secondes permute les deux groupes ainsi classés en faisant passer les Cézanne devant les deux peintures traditionnelles (à l'avantage, tout particulièrement, du Tb22, Portrait de Madame Cézanne, qui a arrêté 89\% des visiteurs).

On peut saisir sans doute, grâce à cette permutation de rangs portant sur deux ensembles de tableaux iconographiquement et iconologiquement contrastés, le sens différent de ces deux indicateurs: le nombre d'arrêts semble enregistrer un effet mécanique de politesse envers la notoriété des toiles et des noms propres. Il manifeste d'abord et d'une manière qu'on pourrait dire «réflexe» l'entrée en jeu, au moins négative, d'une norme qui exclut, comme un "raté» d'interaction sociale (même pratiquée silencieusement dans un espace de musée), l'impolitesse que représenterait, en situation publique, le refus d'un arrêt de salutation. Une fois l'arrêt devant le tableau obtenu, le temps plus ou moins long pendant lequel 
le visionnement se trouve mis au service des actes d'exploration qui composent une expérience esthétique ou, à tout le moins, une curiosité perceptive, a chance de mesurer plus et autre chose, même si on ne peut identifier par l'observation le contenu sémique de l'expérience qui se déroule pendant le temps de visionnement. Le temps de contemplation que l'on consacre à un tableau ou à une image échappe en effet plus complètement à la conscience que l'on en prend ou à la conscience de l'interaction sociale en cours que la décision, plus ostentatoire et plus socialisée, d'arrêter un instant la déambulation. L'arrêt fonctionne un peu comme un coup de chapeau donné à une ouvre qu'on croise en public: il arrive donc assez généralement que le geste de politesse culturelle se suffise à lui-même, tandis que l'immobilisation aux fins de contemplation, qui n'engage pas au même degré une transaction publique sur l'image de soi, a chance de révéler davantage des plaisirs, des perplexités ou des intérêts inhérents à une perception artistique.

Cette opposition se voit clairement dans notre enquête parce qu'elle est illustrée paradigmatiquement par la permutation des tableaux 9 et 5 et des tableaux 22, 21, 23 dans les deux classements. Le classement par le nombre d'arrêts, qui signale le coup de chapeau quasi obligatoire, est très proche du classement que formuleraient les visiteurs s'ils répondaient à un questionnaire de préférences. C'est bien en effet ce classement que suggère la réponse à la seule question de préférence posée directement par l'enquêteur en fin d'observation et à laquelle les sujets ont répondu en classant majoritairement «les Cézanne» avant tous les autres parmi les tableaux qu'ils ont préférés dans les salles A et $\mathrm{C}^{19}$ - exactement comme ils l'auraient fait dans un questionnaire, en anticipant le contrôle de légitimité (supposé) par le questionneur.

Bref, les Cézanne ont un classement qui les privilégie systématiquement par référence à des indicateurs de comportement superficiel ou verbal plus que par des caractéristiques de l'exploration visuelle, comme celle que mesure le temps de visionnement. Cela vaut pour les trois Cézanne les plus remarqués, mais également pour tous les autres. On peut ainsi confirmer et préciser le sens contrasté des deux indicateurs. Nos résultats suggèrent qu'ils isolent tendanciellement deux composantes distinctes de l'expérience de la peinture qui sont inégalement représentées dans les tableaux soumis à l'enquête. Il faut pour l'apercevoir systématiser la comparaison des rangs en construisant un tableau qui isole les plus fortes permutations de classement. On voit alors que tous les Cézanne qui bougent de plus de 10 rangs d'un classement à l'autre sont systématiquement mieux classés par le nombre d'arrêts que par le temps moyen de visionnement.

B. Que signifie le classement opéré par la lecture des notices C'est en classant les 32 tableaux par la lecture des notices les concernant qu'apparaissent les plus fortes permutations par rapport aux classements que nous avons examinés jusqu'ici. Et d'abord on voit se détacher au premier rang le Tb27 (La Vallée de Saint-Pons, de Joseph Gibert) dont $41 \%$ des visiteurs ont lu la notice, précisant que le peintre fut le maître de Cézanne à l'École de dessin d'Aix, alors que ce tableau figurait au $27^{\mathrm{e}}$ rang par le temps de visionnement. Associé à deux petits paysages peints dans la jeunesse de Cézanne (À la tour de César et Paysage), il est présenté avec eux sur un panneau et pose par là explicitement au regard du visiteur la question de comprendre ce rapprochement muséologique. C'est la notice du Tb27 qui donne la réponse, car elle isole la curiosité de type historique qui s'est portée sur trois tableautins qui

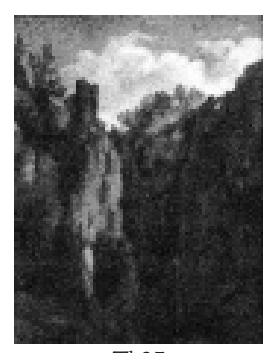

Tb27

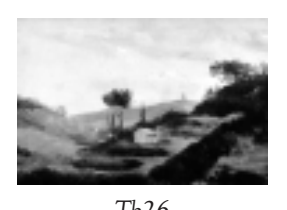

Tb26 ont été parmi les moins longtemps regardés, tant par le temps brut que par le temps pondéré.

Le sort fait à ce petit panneau comparatiste isole clairement, par l'intérêt exclusif accordé à la prise d'information (qui se manifeste dans la lecture fréquente de la notice comme dans le nombre d'arrêts où le Tb27 figure au cinquième rang), la composante ascétique sinon érudite présente en tout intérêt artistique, et souvent 
même dissociée ou hypertrophiée par rapport au plaisir sensoriel ou au retentissement affectif. On peut même supposer que c'est ici une dimension encore plus particulière de la curiosité historiographique portée à l'art qui se manifeste, celle qui privilégie dans l'attirance exercée par le personnage charismatique de l'artiste, sa jeunesse ou sa précocité: qu'était donc Cézanne avant d'être vraiment Cézanne? quel débutant a-t-il été? son génie s'annonçait-il? Kris et Kurz ont montré, dans leur prospection des représentations sociales de l'artiste au travers des cultures, la richesse des investissements qui s'attachent à l'enfance du créateur ou aux promesses de son génie 20 .

La lecture systématique des permutations dans les classements (entre lecture des notices et temps dépensé face à chaque tableau), comme l'analyse comparée des lectures prolongées ou rapides des notices permettent d'isoler mieux encore la composante ascétique de la réception révélée par les classements contrastés du Tb27 en fonction de nos différents indices $^{21}$.

\section{Retourner devant un tableau}

Le retour devant un tableau relève d'une forme de déambulation dans le musée qui caractérise assez bien certains groupes de visiteurs et qui, par sa dissociation avec les attitudes liées au métier et au diplôme, signale l'existence d'une sorte de code muséal ou qui s'apparente, si l'on préfère, à la maîtrise des «bonnes manières de visiter un musée». Mais cette propension liée aux attitudes dont sont génériquement porteurs certains groupes de visiteurs s'accomplit-elle indifféremment quel que soit le tableau? Ou bien certains tableaux révèlent-ils par là même quelque chose de leur effet propre? L'enquête n'a pas permis de trancher clairement sur ce point, même s'il apparaît que, dans l'ensemble, le retour est sans doute l'indicateur le plus déroutant parmi ceux que nous avons utilisés.

Le classement des tableaux par leur nombre de retours se signale d'abord par de faibles fréquences - même les trois Cézanne emblématiques n'ont ramené pour un deuxième regard que $12 \%$ de

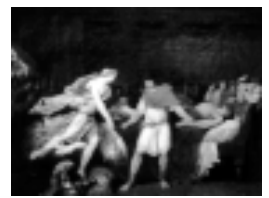

$\mathrm{Tb} 4$ visiteurs. Le sort fait au Tb4 (Thétis et Achille à la mort de Patrocle par Drouais) est singulier en ce qu'il a été le fait de $30 \%$ de retours des visiteurs, alors qu'il s'agit là d'un tableau peu singularisé par les autres comportements muséaux (il figure toujours en milieu de classement). On pourrait conjecturer qu'est intervenu ici un effet de disposition muséologique si l'on se reporte à la localisation de ce tableau: il pourrait avoir été omis dans le premier moment des déambulations avant de bénéficier d'un repentir. Mais comme le handicap muséologique tenant à sa position est faible, il pourrait tout aussi bien s'agir d'un aspect spécifique de l'effet-tableau. Ce serait beaucoup plus intéressant mais la chose reste ici hypothétique. Interroger son iconographie ou sa facture ne deviendrait possible que si un tel effet était attesté sur une série et non sur un cas: seuls de plus vastes échantillons d'offres pourraient répondre à la question de la spécificité éventuelle d'un tel indicateur.

\section{L'EFFET-TABLEAU ET LES ATTITUDES DES VISITEURS: \\ la caractérisation des tableaux par les groupes \\ et des groupes par les tableaux \\ Tout le monde admet que l'on puisse caractériser} des groupes par leurs choix ou préférences, en matière d'art comme ailleurs. Il est plus difficile d'admettre que le sort fait à des œuvres par des récepteurs réels fasse partie intégrante du sens de ces œuvres. Mais c'est précisément le propre d'une sociologie de la réception que de se refuser à caractériser des œuvres d'art par la seule analyse interne de leur structure ou de leur sens, indépendamment du traitement que leur réservent des publics réels, dans la mesure où la lecture qu'ils font des œuvres est attestée par l'observation.

1. Préférences et dédains dans les différents groupes: aucun groupe n'est "puriste»

L'enquête dont les résultats sont présentés ici avait précisément pour visée d'utiliser les données quantitatives recueillies sur le traitement réservé à des 
tableaux de musée par un public de visiteurs, afin de caractériser réciproquement les groupes de visiteurs par les tableaux que leur comportement privilégie ou néglige, et les groupes de tableaux qui sont rapprochés par les choix ou les dédains propres à différents groupes de visiteurs.

Pour ce faire, après avoir commenté les préférences et les dédains que manifestent les choix absolus des différents groupes (A), on entreprendra de serrer de plus près la personnalité différentielle tant des groupes de visiteurs que des groupes de tableaux. Nous négligerons ici les résultats obtenus à partir d'analyseurs plus complexes qui ont pour effet de neutraliser, dans les choix de chaque groupe, les choix qu'ils partagent avec d'autres et dont la convergence produit les choix majoritaires qu'enregistre le classement général des 32 tableaux sur l'ensemble de l'échantillon. Nous nous contenterons de présenter in fine la synthèse de ces résultats en commentant la façon dont les visiteurs de notre échantillon catégorisent les genres de peinture par leurs comportements sémiques (B).

Tableau 4

Les six tableaux les plus regardés* (temps moyens «bruts» et arrêts) par groupes et dans l'ordre de leur classement

\begin{tabular}{|c|c|c|}
\hline & TABLEAUX REGARDÉS LE PLUS LONGUEMENT & TABLEAUX AYANT PROVOQUÉ LE PLUS D’ARRÊTS (4 PREMIERS RANGS) \\
\hline Professionnels & $\begin{array}{c}\text { 21(Cézanne)/23(Cézanne)/22(Cézanne)/9(Revoil)/ } \\
\text { 5(Granet)/24(Cézanne) }\end{array}$ & $\begin{array}{l}\text { 22(Cézanne)/21(Cézanne)/23(Cézanne)/ } \\
\text { 5-9-20-28(Granet, Revoil, Cézanne,Drolling) }\end{array}$ \\
\hline Professions intellectuelles & $\begin{array}{c}\text { 9(Revoil)/5(Granet)/15(Le Nain)/22(Cézanne)/ } \\
\text { 17(Granet)/21(Cézanne) }\end{array}$ & $\begin{array}{l}\text { 21-23(Cézanne)/20-24-31(Cézanne)/ } \\
\text { 5-9-22-28(Granet,Revoil,Cézanne,Drolling) }\end{array}$ \\
\hline "Autres" & $\begin{array}{c}\text { 9(Revoil)/5(Granet)/15(Le Nain)/22(Cézanne)/ } \\
\text { 17Granet)/21(Cézanne) }\end{array}$ & $\begin{array}{l}\text { 22(Cézanne)/5-27(Granet,Gibert)/ } \\
\text { 9-31(Revoil,Cézanne)/21-23(Cézanne) }\end{array}$ \\
\hline Milieu social inférieur & $\begin{array}{l}5(\text { Granet }) / 9(\text { Revoil }) / 13 \text { (Bernard)/ } \\
14(\text { Van Beest }) / 15(\text { Le Nain) } / 18 \text { (Teniers) }\end{array}$ & $\begin{array}{l}\text { 22-28-29-5(Cézanne,Drolling,École F.XVII,Granet)/11-13-14-16-20-23-27-31 } \\
\text { (Géricault,Bernard,Van Beest, Ryckaert,Cézanne,Cézanne,Gibert,Cézanne) }\end{array}$ \\
\hline Milieu social moyen & $\begin{array}{c}\text { 9(Revoil)/5(Granet)/21(Cézanne)/15(Le Nain)/ } \\
\text { 23(Cézanne)/22(Cézanne) }\end{array}$ & $\begin{array}{l}\text { 22(Cézanne)/21-23-5(Cézanne,Cézanne,Granet)/ } \\
\text { 31(Cézanne)/9-19(Revoil,Cézanne) }\end{array}$ \\
\hline Milieu social supérieur & $\begin{array}{l}\text { 9(Revoil)/22(Cézanne)/5(Granet)/23(Cézanne)/ } \\
\text { 21(Cézanne)/15(Le Nain) }\end{array}$ & $\begin{array}{l}\text { 22(Cézanne)/21-27-9(Cézanne,Gibert,Revoil)/ } \\
\text { 20(Cézanne)/23(Cézanne) }\end{array}$ \\
\hline Diplôme inférieur & $\begin{array}{c}\text { 5(Granet)/9(Revoil)/15(Le Nain)/14(Van Beest)/ } \\
\text { 13(Bernard)/17(Granet) }\end{array}$ & $\begin{array}{l}\text { 22(Cézanne)/27-31(Gibert,Cézanne)/3-5(Granet)/ } \\
\text { 14-16-19-23(Van Beest,Ryckaert,Cézanne,Cézanne) }\end{array}$ \\
\hline Diplôme moyen & $\begin{array}{c}\text { 9(Revoil)/5(Granet)/21(Cézanne)/22(Cézanne)/ } \\
\text { 23(Cézanne)/15(Le Nain) }\end{array}$ & $\begin{array}{l}\text { 22(Cézanne)/21(Cézanne)/23(Cézanne)/ } \\
\text { 5(Granet)/20-31(Cézanne) }\end{array}$ \\
\hline Diplôme supérieur & $\begin{array}{l}\text { 9-22(Cézanne) } / 5(\text { Granet }) / 23(\text { Cézanne }) / \\
\text { 21(Cézanne) } / 24(\text { Cézanne })\end{array}$ & $\begin{array}{c}\text { 22(Cézanne)/9(Revoil)/21-27(Cézanne,Gibert)/ } \\
\text { 5-23(Granet,Cézanne) }\end{array}$ \\
\hline $18-32$ ans & $\begin{array}{l}\text { 9(Revoil)/22(Cézanne)/23(Cézanne)/31(Cézanne)/ } \\
\text { 21(Cézanne)/15(Le Nain)/21(Cézanne) }\end{array}$ & $\begin{array}{l}\text { 22(Cézanne)/21(Cézanne)/31(Cézanne)/ } \\
\text { 23(Cézanne)/20(Cézanne) }\end{array}$ \\
\hline $33-47$ ans & $\begin{array}{c}\text { 9(Revoil)/22(Cézanne) } / 5 \text { (Granet) } / 21 \text { (Cézanne) } / 15(\text { Le } \\
\text { Nain)/31(Cézanne)/17(Granet) }\end{array}$ & $\begin{array}{l}\text { 22(Cézanne)/5(Granet)/9-27(Revoil,Gibert)/ } \\
\text { 21-23(Cézanne)/20(Cézanne) }\end{array}$ \\
\hline 48 ans et plus & $\begin{array}{l}\text { 9(Revoil)/5(Granet)/31(Cézanne)/15(Le Nain)/ } \\
\text { 23(Cézanne)/16(Ryckaert)/21(Cézanne) }\end{array}$ & $\begin{array}{l}\text { 22(Cézanne)/16(Ryckaert)/5-9-10-19-23-27(Granet,Revoil, } \\
\text { Géricault,Cézanne, Cézanne,Gibert)/6-14(David,Van Beest) }\end{array}$ \\
\hline $\begin{array}{l}\text { Rappel du classement } \\
\text { sur l'échantillon }\end{array}$ & $\begin{array}{c}\text { 9(Revoil)/5(Granet)/22(Cézanne)/21(Cézanne)/ } \\
\text { 23(Cézanne)/15(Le Nain) }\end{array}$ & 22(Cézanne)/21(Cézanne)/23-5(Cézanne,Granet)/27(Gibert)/9(Revoil) \\
\hline
\end{tabular}

* Le tiret indique les tableaux classés ex aequo par l'indicateur 
À première vue la monotonie des têtes de classement dans les différents groupes n'est rompue que par deux permutations systématiques:

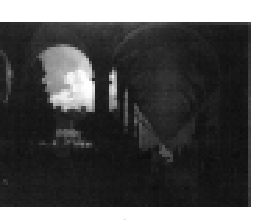

$\mathrm{Tb5}$

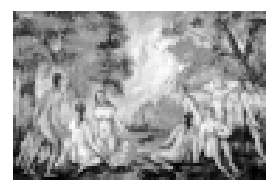

Tb21

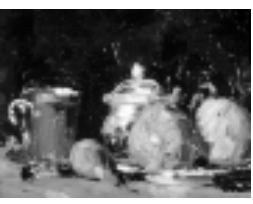

Tb23

- l'une dont nous avons anticipé

le commentaire afin de faire

ressortir la spécificité du

comportement des professionnels

qui sont les seuls à classer trois

Cézanne (Tb21, Tb23, Tb22)

avant le couple vedette du

classement général formé par le

Revoil et le Granet (Tb9 et Tb5);

- l'autre qui oppose la structure

des classements au temps à la

structure des classements au

nombre d'arrêts. Les Cézanne,

dont nous venons de voir qu'ils

étaient toujours mieux classés, sur

l'ensemble de l'échantillon, aux

arrêts qu'aux temps - classements

rappelés ici en bas de tableau - conservent

tendanciellement cette caractéristique déambulatoire

dans les classements des divers groupes.

Une singularité ne s'attache dans ces têtes de classement qu'à deux groupes - et à vrai dire ils sont très proches statistiquement, puisqu'il s'agit du milieu social inférieur et des diplômés les plus bas -, c'est la disparition dans ces deux cas de tout Cézanne dans les dix premiers rangs. On voit que, lorsqu'ils ne sont pas sous l'influence d'une interrogation par l'interview ou le questionnaire - qui met toujours en jeu le caractère légitimant ou illégitimant des réponses -, les groupes populaires manifestent franchement, par le temps de visionnement qu'ils leur consacrent, une assez totale indifférence au plaisir visuel pris à des Cézanne, pourtant fléchés dans ce musée par la réputation ou les dépliants. Mais, on le voit aussitôt, les Cézanne réapparaissent, pour ces groupes, en tête de leurs classements pour le nombre d'arrêts: on a ici la confirmation de l'interprétation que nous proposions plus haut de cet indicateur qui signale toujours l'existence d'une forte composante de conformité sociale; l'arrêt devant un tableau, dans l'espace social

du musée, constitue un indicateur direct de la sensibilité à la notoriété, dès lors qu'il fonctionne, pour un groupe, à l'inverse du temps donné à un tableau.

Il reste que la réapparition, dans le classement opéré par chaque groupe, de l'essentiel du classement général observable sur l'ensemble de l'échantillon constitue un résultat qui signifie quelque chose: le goût général, n'est pas, dans un public de musée, la simple résultante arithmétique de goûts totalement divergents propres à chaque groupe; il y a bien en chaque groupe une juxtaposition, à peu près identique, de goûts pour des tableaux qui sont, eux, extrêmement différents par leur texture iconographique ou leur facture formelle. Le purisme ou l'exclusivisme du goût pictural, souvent revendiqué comme révélateur d'une «vraie» expérience esthétique, ne se laisse déceler statistiquement dans aucun groupe. Et, encore une fois, ce résultat que trahissent les comportements, à la fois objectifs et naïs de la visite d'un musée, se dissimulerait sans doute derrière des réponses plus convenues à des questionnaires 22 .

\section{Deux formes de "l'effet-tableau»}

On peut aller au-delà des données absolues de la mesure - ici des temps bruts de visionnement ou des nombres d'arrêts - en mettant en rapport les préférences et les dédains qui caractérisent le plus précisément chaque groupe socioculturel. En effet, l'existence, dans chaque groupe, d'un goût dominant et majoritaire (même si ce goût dominant est luimême bariolé) ne doit pas faire passer à l'arrière-plan les divergences des systèmes préférences/dédains qui permettent de cerner de plus près la physionomie de chaque tableau ou de chaque groupe socioculturel. Un goût ne se décrit pas seulement dans les plaisirs qu'il recherche, mais aussi dans ses indifférences.

On a ci-dessus caractérisé l'effet propre à un tableau comme un effet qui serait, à la limite, capable de s'imposer à l'attention des spectateurs, quels que soient les groupes socioculturels auxquels ils appartiennent, c'est-à-dire indépendamment des prédispositions culturelles socialement conditionnées 
dont les visiteurs sont génériquement et

différentiellement porteurs. On peut, en neutralisant les choix majoritaires, isoler la forme consensuelle ou unanimiste d'un effet-tableau. Il suffit de se demander quelles sont les toiles bien classées par l'ensemble de l'échantillon (sur le Tableau 2) qui disparaissent du haut d'un classement qui les reclasserait par la variation de leur pouvoir de faire réagir différemment les groupes de l'échantillon. Autrement dit, quelles sont celles des sept toiles classées en tête sur le Tableau 2 qui ne figurent plus dans les onze caractérisées par une forte somme de réactions spécifiant et distinguant les groupes ${ }^{23}$ ? Ce sont le Tb9 (le Revoil qui était la vedette incontestée du classement aux temps «bruts»), le Tb5 (la toile de Granet qui le suivait au classement) et le Tb15 (Le Nain classé lui aussi au-dessus du temps moyen par tableau de l'ensemble de l'échantillon).

Si un effet-tableau, ainsi défini en sa forme consensuelle, se laisse bien déceler sur cette enquête, on voit qu'il ne concerne pas les Cézanne, mais des tableaux dont nous avons déjà commenté quelquesunes des caractéristiques picturales qui semblaient appeler une attention largement répartie dans tous les groupes ou, ce qui revient au même, n'appelaient presque jamais le dédain. Mais il faut alors isoler une autre forme de l'effet-tableau, qu'illustrent ici plusieurs Cézanne: quelques toiles de ce peintre figurant dans les sept premiers sur le Tableau 2 doivent en effet cette position à une tout autre répartition des attentions et des dédains dans les différents groupes: ce sont le Tb22 (3e), le Tb21 (5e) et le Tb23 (6e). L'effet-tableau qui caractérise ces Cézanne associe un bon classement sur l'ensemble de l'échantillon à un fort pouvoir de produire des réactions contrastées selon les groupes, puisque leur pouvoir réactif est parmi les plus élevés et que ce pouvoir réactif est nettement contrasté: les préférences spécifiques de certains groupes pour le Tb22, le Tb21 et le Tb23 sont associées à des dédains spécifiques d'autres groupes pour ces mêmes tableaux.

Divers traitements permettent d'isoler les dix toiles qui séparent le mieux les différents groupes. Le portrait de Bernard (Tb13) avec 4 préférences et
7 dédains est le tableau le plus disputé entre les 16 groupes que distingue l'enquête. On peut dire qu'il illustre le mieux l'effet-séparateur d'un tableau, en entendant par là le contraire de l'effet-consensuel d'un tableau tel que l'illustrent le Revoil et le Granet. Mais certains tableaux, comme les Tb21, 22 et 23 de Cézanne, associent, comme nous venons de le voir, ces deux effets caractéristiques de signification inverse: l'association de ces deux composantes de la réception peut ainsi être retenue comme un indicateur du pouvoir d'un tableau de provoquer une réaction complexe. Sur les dix tableaux isolés par leur effet-séparateur, cinq sont des toiles de Cézanne (Les Baigneuses, le Portrait de Madame Cézanne, la Bethsabée au bain, l'Apothéose de Delacroix et la Nature morte), mais seules trois d'entre elles (Tb21, 22, 23) présentent la forme mixte de l'effet-tableau (effet paradoxal, à la fois unanimiste et séparateur) telle que nous venons de la définir. Les indicateurs de la réception dont nous dégageons progressivement le sens mesurent en effet, dans ce cas, la coexistence ponctuelle de la recherche d'une originalité et de l'alignement du goût.

\section{La catégorisation des genres par leur réception:}

les convergences statistiques $d u$ comportement perceptif

On peut, pour conclure synoptiquement, recourir au traitement systématique des relations entre données que permet l'analyse factorielle des correspondances.

Quelques plans factoriels d'une analyse des correspondances

confirment les relations que nous avons commentées avec le plus

d'insistance: elles sont très proches des données récapitulatives

que nous avons utilisées pour neutraliser la tendance majoritaire. L'analyse factorielle est en effet fondée sur un principe analogue de neutralisation. On se contentera de donner ici la forme simplifiée que le calcul des traces 24 retient de l'analyse des correspondances. Ce programme visualise directement la hiérarchie des corrélations les plus fortes jusqu'à un plancher 25 . On y lit donc les degrés de saturation des relations entre les choix de tableaux (mesurés au temps de visionnement) et les groupes de visiteurs qui ont le plus contribué à cette saturation. L'ordre hiérarchique dans lequel apparaissent graphiquement les traces est résumé dans le tableau qui figure ici les trois phases (1, 2 et 3) par la taille des noms de modalités. (Voir page suivante.) 
Schéma 3

Tracé des relations entre les groupes et les tableaux préférés ou dédaignés dans l'espace de l'analyse des correspondances

+ Prétérence $\approx$ Indifférence $\quad-$ Dédain
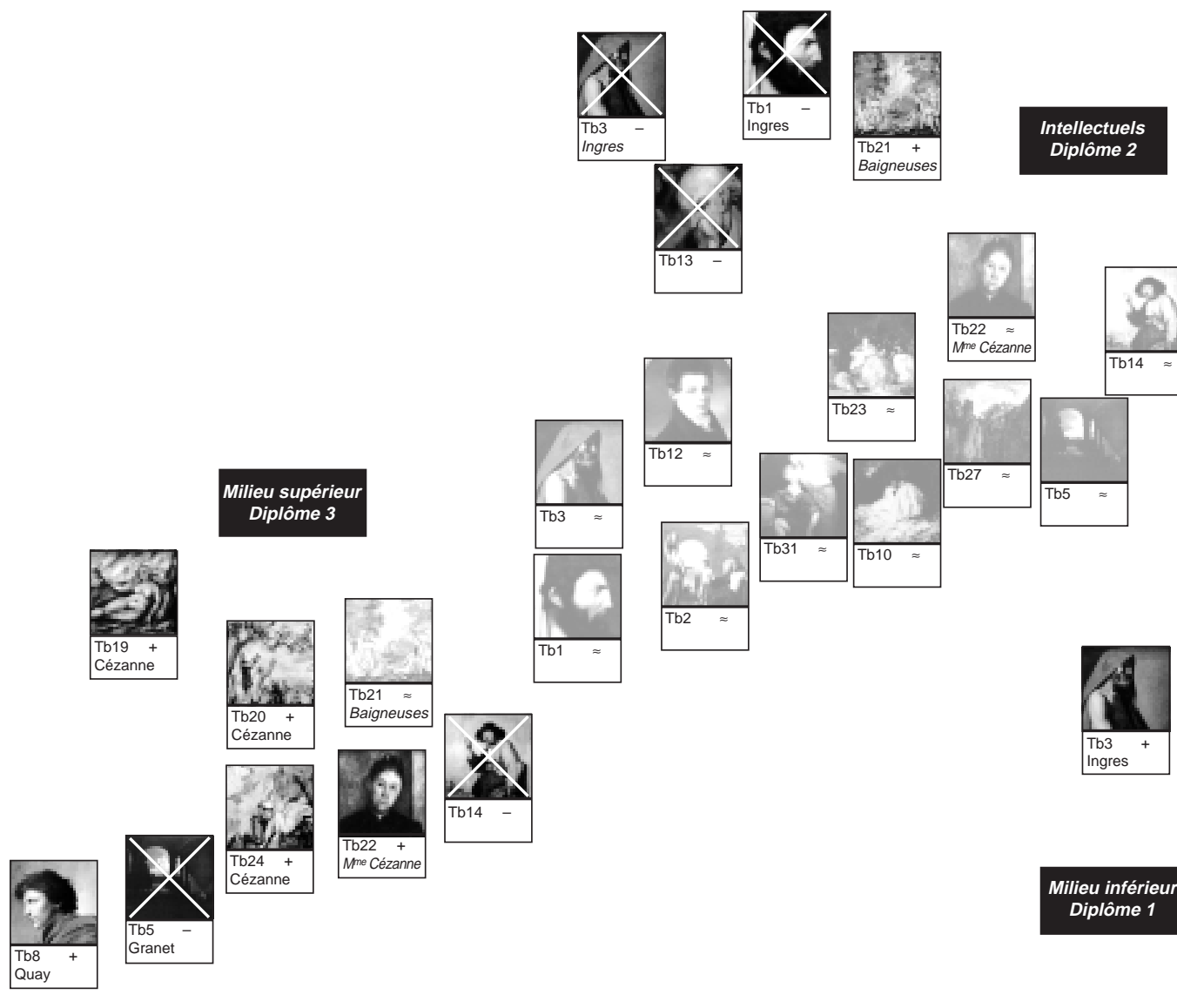

Diplôme 2
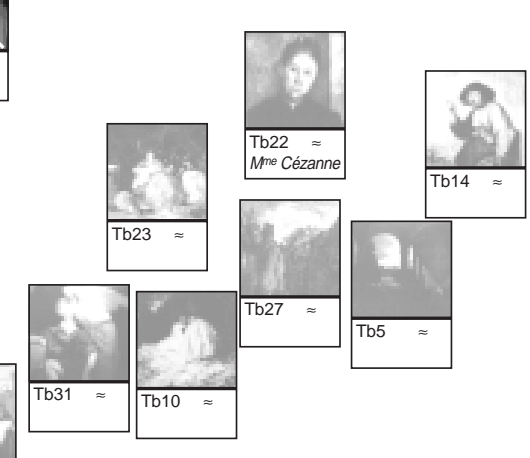

$1^{\circ}$ - Le groupe de tableaux qui est le premier constitué par l'analyse des «traces» - parce que les valeurs numériques des corrélations qui le
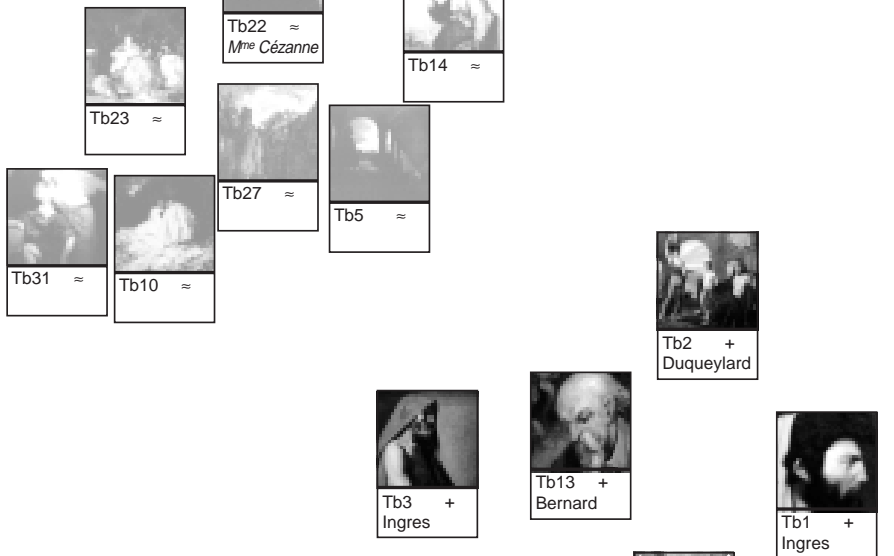

construisent en sont les plus fortes - est celui qui rapproche les préférences pour trois portraits (Étude d'homme de profil et Étude d'homme, assis de profil d'Ingres, et Hommage à Cézanne de Bernard) et une scène à l'antique (Bélisaire et son guide de Duqueylard). Ces préférences relient les groupes qui ont le plus contribué à leur conjonction: ce sont les diplômés inférieurs, le milieu social inférieur et le groupe des plus âgés. Aux préférences qui les rapprochent sont

associés les rejets des trois Cézanne les plus connus (Portrait de Madame Cézanne, Les Baigneuses et Nature morte).

$2^{\circ}$ - Le groupe de tableaux qui se constitue en second est celui qui rapproche les préférences pour un portrait de Quay (Tête d'étude), et les préférences pour quatre Cézanne (Portrait de Madame Cézanne, Femme nue au miroir, Bethsabée, Apothéose de Delacroix). Cette constellation de préférences relie les visiteurs de milieu social supérieur et les diplômés ayant fait les études supérieures les plus longues (plus de quatre ans après le bac). Aux préférences qui rapprochent ces 
groupes sont associés aussi bien le dédain pour le Granet, qui triomphait sur l'ensemble de l'échantillon, et pour un portrait de Van Beest (Homme à la pipe), que l'indifférence aux Baigneuses de Cézanne.

$3^{\circ}$ - Le groupe qui se constitue en dernier, c'est-à-dire dont la consistance statistique est la plus faible, est celui qui rapproche la préférence pour Les Baigneuses et pour ce seul tableau - du dédain des deux toiles d'Ingres (Étude d'homme de profil et Étude d'homme, assis de profil) et du portrait de Bernard (Hommage à Cézanne) ainsi que de toute une série d'indifférences relatives: Portrait de Madame Cézanne, Nature morte, Homme à la pipe (Van Beest), Vallée de Saint-Pons (Gibert), Le Cloître de la chartreuse de Sainte-Marie-desAnges (Granet), Portrait du Comte Henri Simeon (Kinson), Oriental assis sur un rocher (Géricault). Cette configuration de goûts, de dédains et d'indifférences relatives rapproche alors deux groupes: celui des intellectuels (par opposition aux «professionnels» de l'image ou de la peinture) et celui des diplômés supérieurs de niveau moyen (du baccalauréat à bac +3).

L'intérêt de la technique de calcul utilisée ici, qui ne retient, au travers de l'analyse de toutes les corrélations entre les données, que celles dont la convergence a le plus de volume statistique, est de dégager fortement quelques principes d'une anatomie contrastive des goûts, tels qu'on peut les formuler $a$ posteriori, sans préjuger a priori de l'appartenance des toiles à une catégorie, fût-elle même celle de l'ensemble des tableaux d'un même peintre.

Le calcul montre ainsi que les rejets, voire les indifférences, sont aussi importants pour caractériser une convergence entre des choix de groupes, c'est-àdire un goût structuré, que les préférences les plus fortes. On voit par exemple comment le dédain des Cézanne précise le goût des groupes les moins cultivés pour des portraits ou pour une scène «à l'antique», ou comment le dédain marqué, par les groupes intellectuels ou moyennement diplômés, pour le Granet accentue, dans la deuxième constellation, la recherche d'une position tranchée. Statistiquement, cette caractérisation négative d'un goût par ses évitements pèse lourd, c'est-à-dire $66 \%$ des 21 premières modalités. L'expérience esthétique est d'abord une expérience qui s'affirme dans et par une expérience du plaisir artistique comme indissociable d'un plaisir d'exclusion ou, à la limite, de mépris: seuls quelques rares messages, et eux seuls, sont ressentis et choisis comme des «œuvres» en ceci précisément qu'ils se détachent sur fond du quelconque et du n'importe quoi, signaux eux-mêmes aussi signifiants de l'insipidité perceptive du quotidien: le sentiment du banal exclut d'emblée la possibilité d'une perception artistique ${ }^{26}$.

Dans les regroupements de tableaux qui sont ainsi rassemblés, positivement ou négativement, par les convergences statistiques du comportement perceptif, on retrouve toujours, dispersés entre plusieurs constellations, des tableaux que la catégorisation coutumière ou savante regroupe traditionnellement dans des unités classificatoires qui se trouvent ici éclatées. L'unité apparemment la plus naturelle et la plus installée dans le langage, celle de catégories comme par exemple «le portrait», «les Cézanne», les «scènes littéraires ou mythologiques", ne résiste pas au test auquel les soumet la réaction différentielle d'un public réel face aux tableaux qui les composent. Ainsi l'ensemble des portraits qui figuraient dans les salles $\mathrm{A}$ et $\mathrm{C}$ révèle que le genre "portrait» peut être reçu très différemment $(a)$ lorsque, s'agissant de ceux de Duqueylard ou de Bernard, ils sont associés, dans la constellation 1 (par les groupes les moins diplômés ou les plus âgés), au rejet des trois Cézanne les plus estimés ailleurs, et (b) lorsque, s'agissant d'un portrait de Quay ou du portrait de Madame Cézanne, ils sont associés à d'autres Cézanne, en particulier à ceux représentant des nus, par les visiteurs de milieux sociaux supérieurs et les plus diplômés.

Ainsi, la réception d'un même "genre», d'une même "manière», d'un même "sujet» change de signification lorsqu'elle se trouve associée à d'autres éléments 
distinctifs (préférences, rejets, indifférences, groupes sociaux) dans une constellation qui donne un autre sens - différentiel - à chacun des éléments qu'elle rassemble: les tableaux choisis en commun contribuent à définir le portrait social des groupes qui les choisissent; et corrélativement les groupes qui effectuent les mêmes choix précisent la définition du sens artistique des tableaux qu'ils choisissent ensemble. L'esthétique est une dimension spécifique de la physionomie symbolique des groupes sociaux; la géographie sociale des publics que rassemble un groupe particulier de tableaux fait partie de la définition artistique de chacun de ces tableaux.

Les trois constellations isolées par le «calcul des traces» qui, rappelons-le, ne retient que le noyau le plus significatif d'un ensemble de convergences statistiques, éclairent, on le voit, les associations de tableaux par les groupes sociaux qui les associent. Létroite association de la préférence pour les portraits de genre, ou les portraits d'Ingres au dédain des toiles de Cézanne les plus connues ne prend tout son sens que dans la constellation 1 qui associe à cette orientation du goût les choix des plus bas diplômés, des milieux inférieurs et des plus âgés, dessinant ainsi une nébuleuse du goût polairement opposée au goût cultivé, érudit ou informé. Ceci est sans doute devenu trivial pour la sociologie des consommations culturelles. Mais un autre résultat de l'enquête contredit la représentation d'une hiérarchie unilinéaire des goûts sociaux. Le contraste entre les constellations 2 et 3 montre qu'on simplifie la géographie du goût lorsqu'on oppose le goût populaire à un pôle unique du goût cultivé, entre lesquels se hiérarchiseraient les autres goûts. Ici les choix des visiteurs de milieu supérieur et des plus hauts diplômés construisent une constellation certes "cultivée» mais dont le sens se trouve singularisé par l'abondance des signes d'hétérodoxie. Cette constellation 2 (déjà commentée), qui associe la mise en vedette des Cézanne secondaires ou inattendus au dédain affirmé du trop emblématique Granet, se distingue très bien de la constellation 3 , construite par les choix des «intellectuels» et des diplômés d'enseignement supérieur court: cette dernière est à la fois moins provocante, plus prudente dans son conformisme cultivé et moins sûre de ses hiérarchies. Témoigne de ce goût à la fois scolaire, littéraire et circonspect le poids des "indifférences" relatives à de nombreux tableaux: «indifférences» qui, dans cette enquête, sont signalées par un temps de visionnement moyen plus souvent accordé à un plus grand nombre de toiles. Il est "prudent" - si l'on est prudent culturellement - de ne pas mettre, en visitant un musée, tous ses œufs dans un même panier.

L'absence flagrante des «professionnels» dans cette topographie du goût ne doit pas surprendre: l'analyse se fonde en effet à la fois sur des choix de toiles et sur les groupes qui les choisissent de manière convergente. Les "professionnels de l'art» (au sens où nous avons pu les distinguer dans cette enquête des autres professions «intellectuelles») pratiquent des choix et des comportements eux aussi bien caractérisés statistiquement. Mais comme ils se distinguent par ces choix de tous les autres groupes à la fois, cette singularité même les exclut d'une topographie qui privilégie, par le principe de calcul retenu, la convergence de plusieurs groupes ${ }^{27}$. Leur absence statistique signe l'excentricité (statistique) de leur goût et de leur comportement muséal. Comme on le voit, le "calcul des traces" n'extrait que les conformités sociales qui associent plusieurs conformismes.

Le recours à une méthodologie qui impose de singulariser l'observation de la perception esthétique jusqu'au niveau des tableaux considérés dans leur individualité picturale est évidemment plus exigeant en mesures et en recueil d'informations que le recours à une méthodologie du questionnement des préférences pour des genres. Mais, on le voit, les résultats ne font pas double emploi avec ceux que recueillent des méthodes plus rapides ou plus panoramiques. La mesure sociologique, lorsqu'elle s'applique à des tableaux singuliers et non pas à des catégories prédécoupées par le critique d'art ou le sociologue de questionnaire, révèle d'autres regroupements des toiles et suggère par là une anatomie sociale du goût plus proche des taxinomies 
mises en œuvre dans l'interprétation indigène. Cellesci, souvent déconcertantes, nous apprennent quelque chose des déterminations et des composantes du goût, précisément en ce qu'elles s'écartent des catégorisations savantes. Ce sont ces regroupements, inaccessibles au questionnement opéré selon les catégories habituelles du langage de la description, qui permettent d'approcher la dynamique des expériences artistiques dans leur diversité. C'est dans le rapprochement ainsi opéré a posteriori, par le comportement visuel des spectateurs, entre des tableaux appréhendés dans leur individualité singulière que l'on peut trouver aussi bien le principe de nouvelles questions posées à l'analyse iconographique ou iconologique des œuvres que le principe d'un approfondissement de la description des goûts caractéristiques des groupes sociaux qui ont opéré ces rapprochements.

\section{N O TES}

1. Le présent article est fait à partir du "Temps donné aux tableaux ", compte rendu d'enquête, réalisé en 1991 dans un programme de l'Institut Méditerranéen de Recherche et de Création (Marseille, France). Il en reprend et en élabore les principales conclusions. 2. Pour l'application des principes généraux d'une esthétique de la réception (H.R. Jauss, Pour une esthétique de la réception, Paris, Gallimard, 1973) à un art non verbal comme la peinture, cf. J.-C. Passeron, «L'usage faible des images: enquêtes sur la réception de la peinture", dans Le Raisonnement sociologique, l'espace non poppérien du raisonnement naturel, Paris, Nathan, 1991, p. 257-88; pour la musique, cf. E. Pedler, Sociologie de l'opéra, Parenthèses, 1999.

3. Cf. pour un recensement de ces travaux, H. Gottesdiener, Évaluer l'exposition, Paris, La Documentation française, 1987. L'intention dominante de ces enquêtes est muséologique, portant sur des caractéristiques du dispositif d'offre ou sur les parcours de visiteurs plus que sur la forme et le contenu iconiques des objets offerts: par exemple, A. Going, G. Grifenhagen, «Psychological Studies of Museum and Exhibits at the U. S. National Museum ", The Museologist, 1957, n 64 ; ou A. Gordon, "The Exploration Route in an Exhibition: a New Follow-up Technique Employed in the Ruth Youth Wing", The Israël Museum Journal, 1982, n 1 ; ou encore: H. Gottesdiener, "Analyse de l'influence de l'organisation spatiale d'une exposition sur le comportement des visiteurs", Compte rendu, Nanterre, Laboratoire de Psychologie, 1979. Le perfectionnement technique des mesures va souvent de pair avec l'indifférence à l'égard du sens des gestes et des comportements perceptifs, comme on le voit dans une enquête raffinant, grâce à un système électrique implanté dans le sol, l'enregistrement des pas: R. B. Bechtel, «Hodometer Research in Museums", Museum News, 1967.

4. On verra effectivement dans les pages qui suivent que le sort différent fait aux tableaux de Cézanne les plus regardés (soit par le classement au nombre d'arrêts soit par le classement à la longueur du visionnement) permet de séparer deux visées du regard porté sur une œuvre d'art exposée en public.

5. L. Wittgenstein, Leçons et conversations sur l'esthétique, la psychologie et la croyance religieuse, Paris, Gallimard, 1971.

6. Cf. sur cette phénoménologie minimale des "plaisirs d'art", J.-C. Passeron, Le Raisonnement sociologique..., op. cit., p. 263-264.

7. Dans cette enquête, lorsqu'on demande à l'interviewé devant quel tableau il est resté le plus longuement, il répondait le plus souvent en citant une toile bien connue, alors que la mesure opérée invisiblement par l'enquêteur montrait que ce n'était pas le cas.

8. Voir en annexe la description méthodologique de l'enquête réalisée au musée Granet en 1989.

9. On retrouvera ces analyses, forcément un peu longues, dans le compte rendu intégral de l'enquête publié en 1991.

10. La neutralisation du volume différent des budgets-temps propres à chaque visiteur reste un analyseur utile: elle permet d'observer, lorsque l'on pousse l'analyse jusqu'aux choix caractéristiques des groupes découpés par l'enquête, des particularités qui sont commentées dans la seconde partie de ce texte, lesquelles se trouvent annulées par une mesure globale opérée sur l'ensemble des visiteurs comme celle que nous examinons d'abord.

11. P. Bourdieu et A. Darbel, L'Amour de l'art, Paris, Minuit, 1970. 12. L'observation directe permet de mesurer une dimension de l'attention et de l'intérêt en acte dont les sujets ne sont jamais complètement conscients, comme le montrent les décalages avec les évaluations que recueillent les entretiens ou les questionnaires. Le problème du décalage entre le temps estimé par les pratiquants et le temps réel mesuré par un observateur extérieur à la pratique considérée a été souligné par diverses enquêtes en sociologie de la culture, notamment en ce qui concerne la visite des musées dans L'Amour de l'art. Mais le décalage entre la croissance régulière du temps de visite en fonction du niveau d'éducation et l'absence de variation enregistrée dans les temps estimés était expliqué dans cet ouvrage par l'effort des «sujets les moins cultivés pour se conformer à la norme des pratiques légitimes " (L'Amour de l'art, op. cit., p. 60): l'hypothèse que toute variation culturelle est une «fonction linéaire " du niveau de diplôme simplifie en effet le problème.

13. Ou alors division de ce temps par le temps moyen consacré par chaque sujet aux tableaux qui l'ont retenu plus de trois secondes pour le temps "pondéré».

14. Une enquête sur la «description des tableaux" que nous avons réalisée à la suite de celle dont nous présentons ici les résultats, et qui recourait à l'entretien pour approcher les actes d'exploration et d'interprétation du sens d'une peinture, montrait que la «lisibilité» d'un tableau figuratif (définie par la proportion des «descripteurs théoriquement possibles du tableau " qui était utilisée effectivement dans la description par des visionneurs réels) n'était pas mécaniquement commandée par la richesse du tableau en personnages ou par son ampleur et sa précision figuratives. Ainsi, le vaste panorama portuaire du Lorrain où fourmillaient scènes et personnages ( $L e$ Débarquement de Sainte Paule au port d'Ostie) mobilisait une plus faible part de ses «descripteurs théoriquement possibles» que le Munch qui ne proposait que trois personnages dans un petit paysage (Trois jeunes filles sur un pont). 
15. Les préférences manifestées dans l'enquête sur «La description d'un tableau " par les sujets d'un échantillon tout-venant, qui ont sélectionné dans un lot les reproductions soumises à description détaillée, révélaient une économie comparable du goût majoritaire. Les tableaux les plus dédaignés étaient ceux qui, indépendamment de toute proximité de facture, de style ou d'époque, échappaient le plus complètement à une structure narrative ou aux possibilités de projection affective. Les tableaux préférés constituaient, comme dans la présente enquête, un lot hétérogène (E. Munch, Le Lorrain, La Tour ou Le Breton), dont de nombreuses caractéristiques se laissent rapprocher de celles que nous venons d'analyser dans ce palmarès. Pensons à ce que nous avons appelé «les signes extérieurs de la picturalité » (lumière, grandes compositions), d'ailleurs souvent commentés comme tels par les interviewés à propos du Lorrain comme du La Tour, le pouvoir projectif d'un "motif » à fort retentissement affectif du La Tour, ou les devinettes posées par un scénario implicite (Munch) ou explicite (Le Breton).

16. C'est dans l'enquête spécifiquement dévolue à l'étude des cheminements de l'interprétation d'un tableau, tels qu'ils se manifestent dans les réponses à une invite à « décrire ce que représente un tableau ", que s'est révélée l'importance de ces deux dimensions de l'interprétation d'une peinture.

17. Le succès de la catégorie est chiffré par la moyenne des rangs obtenus par chacun des 32 tableaux dans le temps brut de visionnement: 32 points pour le premier, 31 pour le second, etc. 18. Ce résultat contraste avec ceux qu'ont enregistrés diverses enquêtes sur les préférences iconiques, par exemple celle de E. Bernard (Psycho sociologie du goût en matière de peinture, Paris, Éd. du CNRS, 1973), qui montre, dans un comportement comme celui de l'achat de reproductions, une préférence nette pour les paysages. La divergence est intéressante puisque nous mesurons ici la "préférence" par le temps consenti aux tableaux dans la visite d'un musée. On ne "préfère» pas les mêmes images selon la forme d'appropriation visuelle que définit la situation ou la fonction de la contemplation: la fonction d'ornementation ou de décoration dans laquelle s'investit de manière prédominante l'utilisation de reproductions définit un autre rapport à la peinture que l'usage muséal des tableaux qui réduit le regard au regard sans lendemain, ou, si l'on préfère, le concentre dans l'exaltation du coup d'œil d'exception.

19. Il est caractéristique de l'engendrement mécanique d'un classement par la connaissance sociale de la notoriété que le nom de l'auteur finisse par amalgamer les toiles singulières et fasse à lui seul catégorie perceptive. Dans la préférence majoritaire pour le maitre d'Aix (45\% de l'échantillon), celle-ci s'exprime plus souvent par la nomination "des Cézanne" que par celle de toiles particulières.

20. E. Kris et O. Kurz, L'Image de l'artiste: légende, mythe et magie, Paris/ Marseille, Rivages, 1987.

21. Pour plus de détails, on renvoie ici à l'analyse détaillée présentée dans le compte rendu complet de l'enquête.

22. Pour n'en prendre qu'un exemple, l'indifférence que manifestent par leurs comportements visuels à l'égard des Cézanne les plus faibles diplômés et les visiteurs de milieu populaire n'empêchait pas, sur ce même échantillon, ces sujets de répondre au questionnement placé en fin d'observation qu' «ils avaient préféré les Cézanne» dans la proportion de $44 \%$ (milieu social inférieur) et de $34 \%$ (faibles diplômés), la moyenne de cette réponse dans l'échantillon se situant à $45 \%$

23. Ces mesures de segmentation faisant intervenir les attentions et les dédains sont présentées dans les tableaux du compte rendu d'enquête de 1991.

24. Le programme Traces de P. Cibois, dans sa version de 1989 (MSH, Paris).

25. Le calcul des traces est ici arrêté au seuil de 1,8, c'est-à-dire deux fois moins que la relation la plus forte entre deux modalités du même graphe.

26. Cette composante de l'expérience artistique est articulée à l'ensemble des autres dans Les Sept Plaisirs de l'art de J.-C. Passeron (à paraître).

27. Le recours successif à différentes méthodes de traitement des données se révèle indispensable, non seulement pour les confirmations qu'il procure, mais surtout parce qu'aucune de ces méthodes ne peut, à elle seule, révéler tous les traits distinctifs de comportements que contient virtuellement le matériel d'enquête.

\section{AN N EXE}

Les caractéristiques de l'enquête

(Annexe à laquelle renvoie la note 8 )

La campagne d'observations sur laquelle se fonde l'enquête dont nous présentons ici les résultats a été réalisée au Musée Granet d'Aix-enProvence (France) entre mai et juillet 1987. La période a été choisie afin de tenir compte des variations saisonnières qui affectent la fréquentation des musées. La finalité du protocole, largement orienté par la recherche d'un traitement de statistiques analytiques, était d'enquêter sur les publics les plus diversifiés qui soient. Le prélèvement aléatoire d'entrants sur flux excluait les couples ou les groupes pour des raisons de faisabilité. L'échantillon obtenu $(\mathrm{N}=150)$ était donc représentatif (groupes mis à part) de la fréquentation des visiteurs pour la période considérée. Les principales données démographiques sont les suivantes: femmes (54\%), hommes (46\%); diplômés de l'enseignement supérieur long (bac+4 et au-delà, $38 \%$ ), diplômés de l'enseignement supérieur court (bac à bac $+3,38 \%$ ), diplômés dont le grade est inférieur au bac (24\%); visiteurs français (48\%), visiteurs étrangers (42\%). En ce qui concerne l'appartenance à un milieu social, nous avons préféré cette variable à celle qui identifie les visiteurs par la seule PCS afin de replacer tous les enquêtés, y compris les inactifs, dans leur espace familial. Sont ainsi distingués (1) le milieu inférieur (agriculteurs, ouvriers, artisans et petits commerçants), (2) le milieu moyen (professions intermédiaires, employés) et (3) le milieu supérieur (gros commerçants, industriels, cadres supérieurs et professions libérales). Enfin l'enquête a découpé trois groupes d'appartenance professionnelle en fonction de la proximité au monde des arts plastiques: (1) professions artistiques au sens large (artistes, étudiants et enseignants d'écoles d'art, ou d'autres métiers liés à la manipulation ou au commerce des objets d'art, (2) les autres professions intellectuelles, (3) le reste des professions. Le recueil d'informations était fondé sur l'observation directe. Cette observation n'a pas provoqué d'interférences directes avec les visiteurs, les enquêteurs relevant les indications nécessaires à l'enquête à partir des comportements des visiteurs : parcours de la visite, temps de parcours, déambulations, arrêts devant 32 toiles accrochées dans deux salles du musée. Le bordereau d'observation a identifié divers comportements face à ces toiles (retours, prise de distance ou contemplation rapprochée). Un court entretien effectué à la suite de l'observation a permis d'enregistrer toutes les variables d'état exigées par l'analyse sociologique des résultats. 